TRANSACTIONS OF THE

AMERICAN MATHEMATICAL SOCIETY

Volume 353, Number 10, Pages 3919-3944

S 0002-9947(01)02844-6

Article electronically published on June 6, 2001

\title{
VARIATIONAL PRINCIPLES AND MIXED MULTIFRACTAL SPECTRA
}

\author{
L. BARREIRA AND B. SAUSSOL
}

\begin{abstract}
We establish a "conditional" variational principle, which unifies and extends many results in the multifractal analysis of dynamical systems. Namely, instead of considering several quantities of local nature and studying separately their multifractal spectra we develop a unified approach which allows us to obtain all spectra from a new multifractal spectrum. Using the variational principle we are able to study the regularity of the spectra and the full dimensionality of their irregular sets for several classes of dynamical systems, including the class of maps with upper semi-continuous metric entropy.

Another application of the variational principle is the following. The multifractal analysis of dynamical systems studies multifractal spectra such as the dimension spectrum for pointwise dimensions and the entropy spectrum for local entropies. It has been a standing open problem to effect a similar study for the "mixed" multifractal spectra, such as the dimension spectrum for local entropies and the entropy spectrum for pointwise dimensions. We show that they are analytic for several classes of hyperbolic maps. We also show that these spectra are not necessarily convex, in strong contrast with the "non-mixed" multifractal spectra.
\end{abstract}

\section{INTRODUCTION}

Dimension spectra are one of the primary components of multifractal analysis, a theory developed by physicists and applied mathematicians as a powerful tool for the numerical study of dynamical systems. Dimension spectra encode important information about a dynamical system. Specialists believe that in a number of fundamental situations in dynamics these spectra can be used to fully recover certain aspects of the information contained in the dynamical system, and thus play the role of multifractal moduli, i.e., of complete invariants for dynamical systems obtained solely from their multifractal analysis. As such, multifractal spectra can play a fundamental role in the theory of dynamical systems.

Given a probability measure $\mu$ on a metric space $X$, we define the pointwise dimension of $\mu$ at the point $x \in X$ by

$$
d_{\mu}(x)=\lim _{r \rightarrow 0} \frac{\log \mu(B(x, r))}{\log r}
$$

Received by the editors April 17, 2000 and, in revised form, August 30, 2000.

2000 Mathematics Subject Classification. Primary 37D35, 37C45.

Key words and phrases. Dimension spectrum, entropy spectrum, variational principle.

L. Barreira was partially supported by FCT's Funding Program and NATO grant CRG970161. B. Saussol was partially supported by FCT's Funding Program and by the Center for Mathematical Analysis, Geometry, and Dynamical Systems. 
whenever the limit exists. Here $B(x, r) \subset X$ denotes the ball of radius $r$ centered at $x$. It was recently shown that if $\mu$ is a hyperbolic invariant measure for a $C^{1+\varepsilon}$ diffeomorphism of a compact manifold, then $d_{\mu}(x)$ is well-defined $\mu$-almost everywhere [2]. The dimension spectrum for pointwise dimensions $\mathcal{D}_{D}=\mathcal{D}_{D}^{\mu}$ is defined by

$$
\mathcal{D}_{D}(\alpha)=\operatorname{dim}_{H}\left\{x \in X: d_{\mu}(x)=\alpha\right\},
$$

where $\operatorname{dim}_{H} Z$ denotes the Hausdorff dimension of the set $Z$ (see Section 2.2 for the definition).

Dimension spectra constitute only one class of the general family of multifractal spectra. We now introduce other spectra. Let $f: X \rightarrow X$ be a measurable transformation preserving the measure $\mu$. Consider a finite measurable partition $\xi$ of $X$. We define the $\mu$-local entropy of $f$ at the point $x \in X$ (with respect to $\xi$ ) by

$$
h_{\mu}(f, \xi, x)=\lim _{n \rightarrow \infty}-\frac{1}{n} \log \mu\left(\xi_{n}(x)\right)
$$

whenever the limit exists. Here $\xi_{n}(x)$ is an atom of the partition $\bigvee_{k=0}^{n} f^{-k} \xi$ which contains $x$. By the Shannon-McMillan-Breiman theorem the $\mu$-local entropy is well-defined for $\mu$-almost every $x \in X$. In addition, if $\xi$ is a generating partition of $X$ (i.e., a partition such that $\bigvee_{k=0}^{\infty} f^{-k} \xi$ generates the Borel $\sigma$-algebra of $X$ ) and $\mu$ is ergodic, then $h_{\mu}(f, \xi, x)=h_{\mu}(f)$ for $\mu$-almost every $x \in X$, where $h_{\mu}(f)$ denotes the measure-theoretic entropy of $f$ with respect to $\mu$. The entropy spectrum for local entropies $\mathcal{E}_{E}=\mathcal{E}_{E}^{\mu}$ is defined by

$$
\mathcal{E}_{E}(\alpha)=h\left(f \mid\left\{x \in X: h_{\mu}(f, \xi, x)=\alpha\right\}\right),
$$

where $h(f \mid Z)$ denotes the topological entropy of $f$ on $Z$; we stress that the set $Z$ need not be compact (see Section 2.1 for the definition of topological entropy on arbitrary sets).

If, in addition, $f$ is a differentiable map, one also considers the Lyapunov exponents. Given $x \in X$ we set

$$
\lambda(x)=\lim _{n \rightarrow+\infty} \frac{1}{n} \log \left\|d_{x} f^{n}\right\|
$$

whenever the limit exists. By Kingman's sub-additive ergodic theorem, if $\mu$ is an $f$-invariant probability measure, then $\lambda$ is well-defined $\mu$-almost everywhere. We define the dimension spectrum for Lyapunov exponents by

$$
\mathcal{L}_{D}(\alpha)=\operatorname{dim}_{H}\{x \in X: \lambda(x)=\alpha\},
$$

and the entropy spectrum for Lyapunov exponents by

$$
\mathcal{L}_{E}(\alpha)=h(f \mid\{x \in X: \lambda(x)=\alpha\}) .
$$

It was shown, in a number of situations ubiquitous in dynamics, that the multifractal spectra $\mathcal{D}_{D}, \mathcal{E}_{E}, \mathcal{L}_{D}$, and $\mathcal{L}_{E}$ are real analytic. Moreover, they coincide with the Legendre transform of certain functions characterized in terms of the topological pressure, and thus are always convex (and are in fact "generically" strictly convex with respect to the potential of which $\mu$ is an equilibrium measure). We refer to the book [7] for references and full details.

One can also consider "mixed" multifractal spectra, which intertwine different local characteristics which depend on the dynamics and simultaneously on some other attached structure. This is the case of the pointwise dimensions and the 
local entropies which depend not only on the dynamics but also on the attached measurable structure provided by a given invariant measure.

These "mixed" spectra include the entropy spectrum for pointwise dimensions $\mathcal{D}_{E}=\mathcal{D}_{E}^{\mu}$ defined by

$$
\mathcal{D}_{E}(\alpha)=h\left(f \mid\left\{x \in X: d_{\mu}(x)=\alpha\right\}\right),
$$

and the dimension spectrum for local entropies $\mathcal{E}_{D}=\mathcal{E}_{D}^{\mu}$ defined by

$$
\mathcal{E}_{D}(\alpha)=\operatorname{dim}_{H}\left\{x \in X: h_{\mu}(f, \xi, x)=\alpha\right\} .
$$

We shall refer to these two multifractal spectra as mixed multifractal spectra. It has been a standing open problem in multifractal analysis to describe the spectra $\mathcal{D}_{E}$ and $\mathcal{E}_{D}$; see [7] for an explicit formulation. In particular it has not been known if these spectra are real analytic or if they can be computed by a Legendre transform.

In order to explain why the study of the mixed multifractal spectra is essentially different from the study of the non-mixed spectra, we consider the associated special classes of full measures $\left\{\mu_{\alpha}^{\mathcal{D}}\right\}$ and $\left\{\mu_{\alpha}^{\mathcal{E}}\right\}$. These are invariant ergodic probability measures such that

$$
\operatorname{dim}_{H} \mu_{\alpha}^{\mathcal{D}}=\mathcal{D}_{D}(\alpha) \text { and } \mu_{\alpha}^{\mathcal{D}}\left(\left\{x \in X: d_{\mu}(x)=\alpha\right\}\right)=1,
$$

and

$$
h_{\mu_{\alpha}^{\varepsilon}}(f)=\mathcal{E}_{E}(\alpha) \quad \text { and } \quad \mu_{\alpha}^{\varepsilon}\left(\left\{x \in X: h_{\mu}(f, \xi, x)=\alpha\right\}\right)=1
$$

for each $\alpha$ (see, for example, [7] for details), and play a fundamental role in multifractal analysis. The main difficulty when studying mixed multifractal spectra is that these two families of measures are in general "transversal"; more precisely, they do not satisfy any Fubini-type decomposition, and thus one cannot expect to relate in a simple way mixed and non-mixed spectra. On the other hand it is this transversality that allows us to consider mixed multifractal spectra as further moduli in the study of dynamical systems.

In this paper, for subshifts of finite type and repellers of $C^{1+\varepsilon}$ conformal expanding maps (i.e., expanding maps such that the derivative is a multiple of an isometry at each point), we prove that if $\mu$ is an equilibrium measure with Hölder continuous potential, then:

1. the functions $\mathcal{D}_{E}$ and $\mathcal{E}_{D}$ are real analytic;

2. the functions $\mathcal{D}_{E}$ and $\mathcal{E}_{D}$ are in general not convex, and thus, they cannot be expressed as Legendre transforms.

These statements follow from much more general results and in particular from a new "conditional" variational principle established in this paper. While this variational principle was initially motivated by the study of mixed multifractal spectra, it is in fact of much broader interest. Indeed it unifies and extends a considerable number of results in the multifractal analysis of dynamical systems. Furthermore, it provides a unified approach which allows us to study simultaneously all spectra by using a new multifractal spectrum. In particular, it should be noted that the six spectra in (11)-(6) are only very special cases of the much more general multifractal spectra discussed in this paper.

Instead of formulating general results here, we illustrate our "conditional" variational principle with a rigorous statement in a special case already revealing the richness of the general framework. Given continuous functions $\varphi: X \rightarrow \mathbb{R}$ and 
$\psi: X \rightarrow \mathbb{R}$ with $\psi>0$, we write

$$
K_{\alpha}=\left\{x \in X: \lim _{n \rightarrow \infty} \frac{\sum_{k=0}^{n} \varphi\left(f^{k} x\right)}{\sum_{k=0}^{n} \psi\left(f^{k} x\right)}\right\},
$$

and consider the open interval

$$
I=\left(\inf _{\mu} \frac{\int_{X} \varphi d \mu}{\int_{X} \psi d \mu}, \sup _{\mu} \frac{\int_{X} \varphi d \mu}{\int_{X} \psi d \mu}\right)
$$

where the infimum and supremum are taken over all $f$-invariant probability measures in $X$.

Theorem 1. Let $X$ be a repeller of a topologically mixing $C^{1+\varepsilon}$ expanding map, for some $\varepsilon>0$, and $\varphi, \psi$ Hölder continuous functions in $X$, such that $\psi>0$. If $\alpha \in I$ then

$$
h\left(f \mid K_{\alpha}\right)=\max \left\{h_{\mu}(f): \mu \text { is ergodic and } \frac{\int_{X} \varphi d \mu}{\int_{X} \psi d \mu}=\alpha\right\} .
$$

We refer to the identity in the theorem as a conditional variational principle. Theorem 1 is a particular case of the more general results established below. In fact we obtain a conditional variational principle for the more general class of maps with upper semi-continuous metric entropy, which includes expansive homeomorphisms.

For each pair of functions $\varphi$ and $\psi$, Theorem 1 establishes a conditional variational principle for the corresponding multifractal spectrum $\alpha \mapsto h\left(f \mid K_{\alpha}\right)$. We believe that these spectra can play the role of multifractal moduli. It is an open problem to determine whether a finite number of them is sufficient to fully characterize the dynamics.

In the special case of mixed multifractal spectra we obtain the following new results. For a repeller $X$ of a $C^{1+\varepsilon}$ conformal expanding map $f$, if $\varphi$ is a Hölder continuous function having zero topological pressure and equilibrium measure $\mu$, then

$$
\mathcal{D}_{E}(\alpha)=\sup \left\{h_{\nu}(f): \nu \text { is ergodic and }-\frac{\int_{X} \varphi d \nu}{\int_{X} \log \|d f\| d \nu}=\alpha\right\},
$$

where $h_{\nu}(f)$ is the $\nu$-entropy of $f$, and

$$
\mathcal{E}_{D}(\alpha)=\sup \left\{\operatorname{dim}_{H} \nu: \nu \text { is ergodic and }-\int_{X} \varphi d \nu=\alpha\right\},
$$

where $\operatorname{dim}_{H} \nu=\inf \left\{\operatorname{dim}_{H} Z: \nu(Z)=1\right\}$ is the Hausdorff dimension of the measure $\nu$. In particular:

1. the topological entropy of the set of points for which the pointwise dimension is equal to $\alpha$ can be arbitrarily approximated by the entropy of ergodic measures $\nu$ with $-\int_{X} \varphi d \nu / \int_{X} \log \|d f\| d \nu=\alpha$;

2. the Hausdorff dimension of the set of points for which the local entropy is equal to $\alpha$ can be arbitrarily approximated by the Hausdorff dimension of ergodic measures $\nu$ with $-\int_{X} \varphi d \nu=\alpha$.

By using the variational principles in (7) and (8) we can show that the spectra $\mathcal{D}_{E}$ and $\mathcal{E}_{D}$ are analytic.

The structure of the paper is as follows. In Section 2 we recall several notions from the thermodynamic formalism and dimension theory. Section 3 contains our main results, including a conditional variational principle when the metric entropy 
is upper semi-continuous. The proofs of the results in Section 3 are collected in Section 4 The remaining sections provide applications of this variational principle. In Section 5, we establish the analyticity of the spectra for subshifts of finite type, expanding maps, and hyperbolic diffeomorphisms, and study the associated irregular sets. In Section 6 we obtain new results for expanding maps, including the analyticity of the mixed multifractal spectra, and an explicit example of a non-convex mixed multifractal spectrum. Section 7 provides an application to the multifractal analysis of limit sets of geometric constructions.

\section{TOPOLOGICAL PRESSURE AND NOTIONS FROM DIMENSION THEORY}

2.1. Topological pressure. We recall the notion of topological pressure. Since we need to deal with non-compact sets, we use the notion of topological pressure introduced by Pesin and Pitskel as a Carathéodory characteristic. In particular, the level sets of pointwise dimension and the level sets of local entropy are non-compact virtually for all measures considered in this paper. In fact, in the appropriate sense this is the generic situation (see [3] for details).

Let $f: X \rightarrow X$ be a continuous map of a compact metric space, and $\mathcal{U}$ a finite open cover of $X$. We denote by $\mathcal{W}_{n}(\mathcal{U})$ the collection of words $\mathbf{U}=\left(U_{0}, \ldots, U_{n}\right) \in$ $\mathcal{U}^{n+1}$. For each $\mathbf{U} \in \mathcal{W}_{n}(\mathcal{U})$ we write $m(\mathbf{U})=n$, and define the open set

$$
X(\mathbf{U})=\left\{x \in X: f^{k} x \in U_{k} \text { for } k=0, \ldots, n\right\} .
$$

We say that the collection of words $\Gamma \subset \bigcup_{n \geq 1} \mathcal{W}_{n}(\mathcal{U})$ covers the set $Z \subset X$ provided that $\bigcup_{\mathbf{U} \in \Gamma} X(\mathbf{U}) \supset Z$.

Consider a continuous function $\varphi: X \rightarrow \mathbb{R}$. Given $\mathbf{U} \in \mathcal{W}_{n}(\mathcal{U})$ we write

$$
\varphi(\mathbf{U})= \begin{cases}\sup _{X(\mathbf{U})} \sum_{k=0}^{n-1} \varphi \circ f^{k} & \text { if } X(\mathbf{U}) \neq \emptyset, \\ -\infty & \text { if } X(\mathbf{U})=\emptyset .\end{cases}
$$

For each set $Z \subset X$ and each number $\alpha \in \mathbb{R}$ let

$$
M(Z, \alpha, \varphi, \mathcal{U})=\lim _{n \rightarrow \infty} \inf _{\Gamma} \sum_{\mathbf{U} \in \Gamma} \exp (-\alpha m(\mathbf{U})+\varphi(\mathbf{U})),
$$

where the infimum is taken over all collections of words $\Gamma \subset \bigcup_{k \geq n} \mathcal{W}_{n}(\mathcal{U})$ covering $Z$. Let $\operatorname{diam} \mathcal{U}$ be the diameter of the cover $\mathcal{U}$. One can easily show that the limit

$$
P_{Z}(\varphi) \stackrel{\text { def }}{=} \lim _{\operatorname{diam} \mathcal{U} \rightarrow 0} \inf \{\alpha: M(Z, \alpha, \varphi, \mathcal{U})=0\}
$$

exists, and we call it the topological pressure of $\varphi$ on the set $Z \subset X$ (with respect to $f$ ). We emphasize that $Z$ need not be compact nor $f$-invariant.

When $Z=X$ (and thus $Z$ is compact and $f$-invariant), the number $P_{X}(\varphi)$ coincides with the notion of topological pressure for compact sets introduced by Ruelle in the case of expansive maps, and by Walters in the general case.

When $\varphi=0$, the number

$$
h(f \mid Z) \stackrel{\text { def }}{=} P_{Z}(0)=\lim _{\operatorname{diam} \mathfrak{u} \rightarrow 0} \inf \left\{\alpha: \lim _{n \rightarrow \infty} \inf _{\Gamma} \sum_{\mathbf{U} \in \Gamma} \exp (-\alpha m(\mathbf{U}))=0\right\},
$$

where the infimum in $\Gamma$ is taken over all collections of words $\Gamma \subset \bigcup_{k \geq n} \mathcal{W}_{n}(\mathcal{U})$ covering $Z$, is called topological entropy of $f \mid Z$. This is the definition of topological entropy by Pesin and Pitskel as a Carathéodory characteristic, and coincides with the notion of topological entropy for non-compact sets previously introduced by 
Bowen. Again we emphasize that the set $Z$ need not be compact nor $f$-invariant. When $Z=X$, we recover the classical notion of topological entropy.

We refer to [7, 1] for references and further details.

2.2. Notions from dimension theory. Let $X$ be a separable metric space. Given a set $Z \subset X$ and $\alpha>0$, we define

$$
m(Z, \alpha)=\lim _{\delta \rightarrow 0} \inf _{\mathcal{U}} \sum_{U \in \mathcal{U}}(\operatorname{diam} U)^{\alpha},
$$

where the infimum is taken over all finite or countable cover $\mathcal{U}$ of $Z$ by sets of diameter at most $\delta$. There exists a unique value of $\alpha$ at which $m(Z, \alpha)$ jumps from $+\infty$ to 0 . This value is called Hausdorff dimension of $Z$ and is denoted by $\operatorname{dim}_{H} Z$. We have

$$
\operatorname{dim}_{H} Z=\inf \{\alpha: m(Z, \alpha)=0\} .
$$

For every Borel probability measure $\mu$ on $X$, we define the Hausdorff dimension of $\mu$ by

$$
\operatorname{dim}_{H} \mu=\inf \left\{\operatorname{dim}_{H} Z: \mu(Z)=1\right\} .
$$

We also recall a Carathéodory dimension characteristic introduced by Barreira and Schmeling in [3. We use the notations of Section 2.1.

Let $f: X \rightarrow X$ be a continuous map of a compact metric space, and $\mathcal{U}$ a finite open cover of $X$. Let now $v: X \rightarrow \mathbb{R}$ be a strictly positive continuous function. For each $\mathbf{U} \in \mathcal{W}_{n}(\mathcal{U})$ we define $v(\mathbf{U})$ as in (9).

For each set $Z \subset X$ and each number $\alpha \in \mathbb{R}$, we define

$$
N(Z, \alpha, v, \mathcal{U})=\lim _{n \rightarrow \infty} \inf _{\Gamma} \sum_{\mathbf{U} \in \Gamma} \exp (-\alpha v(\mathbf{U})),
$$

where the infimum is taken over all collections of words $\Gamma \subset \bigcup_{k \geq n} \mathcal{W}_{k}(\mathcal{U})$ covering $Z$. Set

$$
\operatorname{dim}_{v, u} Z=\inf \{\alpha: N(Z, \alpha, v, \mathcal{U})=0\} .
$$

One can easily show that the limit

$$
\operatorname{dim}_{v} Z \stackrel{\text { def }}{=} \lim _{\operatorname{diam} u \rightarrow 0} \operatorname{dim}_{v, u} Z
$$

exists, and we call it the $v$-dimension of $Z$ (with respect to $f$ ).

The following result expresses a relation between the $v$-dimension and the topological pressure, and follows easily from the definitions.

Proposition 2. We have $\operatorname{dim}_{v} Z=\alpha$, where $\alpha$ is the unique root of the equation $P_{Z}(-\alpha v)=0$.

For every Borel probability measure $\mu$ on $X$, let

$$
\operatorname{dim}_{v, u} \mu=\inf \left\{\operatorname{dim}_{v, u} Z: \mu(Z)=1\right\} .
$$

The limit

$$
\operatorname{dim}_{v} \mu \stackrel{\text { def }}{=} \lim _{\operatorname{diam} u \rightarrow 0} \operatorname{dim}_{v, u} \mu
$$

exists, and is called the $v$-dimension of $\mu$.

We are particularly interested in the following examples:

1. If $v=1$, then $\operatorname{dim}_{v} Z=h(f \mid Z)$ for every $Z \subset X$, and $\operatorname{dim}_{v} \mu=h_{\mu}(f)$. 
2. If $v=\log \|d f\|$, where $f$ is a $C^{1+\varepsilon}$ conformal expanding map with repeller $Z$ (see Section [6), then $\operatorname{dim}_{v} Z=\operatorname{dim}_{H} Z$ for every $Z \subset X$, and $\operatorname{dim}_{v} \mu=$ $\operatorname{dim}_{H} \mu$. This follows from the existence of universal constants $c_{1}, c_{2}>0$ (due to bounded distortion) such that

$$
c_{1}(\operatorname{diam} X(\mathbf{U}))^{\alpha} \leq \exp (-\alpha v(\mathbf{U})) \leq c_{2}(\operatorname{diam} X(\mathbf{U}))^{\alpha} .
$$

We also define the lower and upper $v$-pointwise dimensions of $\mu$ at the point $x \in$ $X$ by

$$
\underline{d}_{\mu, v}(x)=\lim _{\operatorname{diam} u \rightarrow 0} \liminf _{n \rightarrow \infty} \inf -\frac{\log \mu(X(\mathbf{U}))}{v(\mathbf{U})}
$$

and

$$
\bar{d}_{\mu, v}(x)=\lim _{\operatorname{diam} u \rightarrow 0} \limsup _{n \rightarrow \infty} \sup -\frac{\log \mu(X(\mathbf{U}))}{v(\mathbf{U})},
$$

where the infimum and supremum are taken over all words $\mathbf{U} \in \mathcal{W}_{n}(\mathcal{U})$ such that $x \in X(\mathbf{U})$.

Proposition 3 ([3]). If $\mu$ is an ergodic $f$-invariant probability measure in $X$, then

$$
\operatorname{dim}_{v} \mu=\lim _{\operatorname{diam} \mathfrak{U} \rightarrow 0} \underline{d}_{\mu, v}(x, \mathcal{U})=\lim _{\operatorname{diam} \mathfrak{u} \rightarrow 0} \bar{d}_{\mu, v}(x, \mathcal{U})=\frac{h_{\mu}(f)}{\int_{X} v d \mu}
$$

for $\mu$-almost every $x \in X$.

Proposition 3 follows from Pesin's theory of Carathéodory characteristics (see [7]), together with the Birkhoff ergodic theorem and the Shannon-McMillanBreiman theorem.

\section{Conditional Variational PRinciple}

3.1. Preliminaries. Let $f: X \rightarrow X$ be a continuous map on the compact metric space $X$. We denote by $C(X)$ the space of continuous functions $\varphi: X \rightarrow \mathbb{R}$. Given functions $\varphi, \psi \in C(X)$ with $\psi>0$, we set

$$
K_{\alpha}=K_{\alpha}(\varphi, \psi)=\left\{x \in X: \lim _{n \rightarrow \infty} \frac{\varphi_{n}(x)}{\psi_{n}(x)}=\alpha\right\}
$$

where

$$
\varphi_{n}(x)=\sum_{k=0}^{n-1} \varphi\left(f^{k} x\right) \quad \text { and } \quad \psi_{n}(x)=\sum_{k=0}^{n-1} \psi\left(f^{k} x\right) .
$$

We denote by $\mathcal{M}(X)$ the set of $f$-invariant Borel probability measures on $X$. Let

$$
\underline{\alpha}=\underline{\alpha}(\varphi, \psi)=\inf \left\{\frac{\int_{X} \varphi d \mu}{\int_{X} \psi d \mu}: \mu \in \mathcal{M}(X)\right\}
$$

and

$$
\bar{\alpha}=\bar{\alpha}(\varphi, \psi)=\sup \left\{\frac{\int_{X} \varphi d \mu}{\int_{X} \psi d \mu}: \mu \in \mathcal{M}(X)\right\} .
$$

Given a positive function $v \in C(X)$ we denote by $\operatorname{dim}_{v} Z$ the $v$-dimension of the set $Z \subset X$ (see Section 2.2 for the definition). For example, when $v=1$ the $v$ dimension of $Z$ coincides with the topological entropy $h(f \mid Z)$. For other examples and for applications see [3]. 
We remark that by considering the case of an arbitrary function $v$, our approach unifies and extends a considerable number of results in the multifractal analysis of dynamical systems. Furthermore, the case of an arbitrary function $v$ is essential to the applications, and in particular in the study of mixed multifractal spectra in Section 6 ]

The function $\mathcal{F}_{v}=\mathcal{F}_{v}^{(\varphi, \psi)}$ defined by

$$
\mathcal{F}_{v}(\alpha)=\operatorname{dim}_{v} K_{\alpha}(\varphi, \psi)
$$

is called the $v$-dimension spectrum for the pair $(\varphi, \psi)$.

We shall first formulate our results in the case of subshifts of finite type. Appropriate generalizations are discussed in Section 3.3. Recall that given a $p \times p$ matrix $A=\left(a_{i j}\right)$ of zeros and ones we consider the set

$$
X=\left\{\left(i_{1} i_{2} \cdots\right) \in\{1, \ldots, p\}^{\mathbb{N}}: a_{i_{n} i_{n+1}=1} \text { for every } n \in \mathbb{N}\right\},
$$

and define the associated subshift of finite type $f: X \rightarrow X$ by $f\left(i_{1} i_{2} \cdots\right)=\left(i_{2} i_{3} \cdots\right)$. The map $f$ is topologically mixing if there exists $k \in \mathbb{N}$ such that $A^{k}$ has only positive entries.

3.2. Conditional variational principle. We denote by $C_{f}(X) \subset C(X)$ the family of continuous functions $\varphi: X \rightarrow \mathbb{R}$ for which there exist $\varepsilon>0$ and $\kappa>0$ such that

$$
\left|\sum_{k=0}^{n-1} \varphi\left(f^{k} x\right)-\sum_{k=0}^{n-1} \varphi\left(f^{k} y\right)\right|<\kappa
$$

whenever $d\left(f^{k} x, f^{k} y\right)<\varepsilon$ for every $k=0, \ldots, n-1$.

Theorem 4. Let $f: X \rightarrow X$ be a topologically mixing subshift of finite type, and $\varphi, \psi, v \in C_{f}(X)$ such that $\psi>0$ and $v>0$. Then the following properties hold:

1. if $\alpha \notin[\underline{\alpha}, \bar{\alpha}]$, then $K_{\alpha}=\emptyset$;

2. if $\alpha \in(\underline{\alpha}, \bar{\alpha})$, then $K_{\alpha} \neq \emptyset$ and

$$
\mathcal{F}_{v}(\alpha)=\max \left\{\frac{h_{\mu}(f)}{\int_{X} v d \mu}: \mu \in \mathcal{M}(X) \text { and } \frac{\int_{X} \varphi d \mu}{\int_{X} \psi d \mu}=\alpha\right\} .
$$

The identity in (10) is a conditional variational principle for the $v$-dimension.

We recall that when $v=1$, we have $\operatorname{dim}_{v} Z=h(f \mid Z)$ for every $Z \subset X$, and $\operatorname{dim}_{v} \mu=h_{\mu}(f)$ for every measure $\mu \in \mathcal{M}(X)$; see Section 2.2 for details. Therefore, taking $v=1$ in Theorem 4 we obtain the following conditional variational principle for the topological entropy.

Theorem 5. Let $f: X \rightarrow X$ be a topologically mixing subshift of finite type, and $\varphi, \psi \in C_{f}(X)$ such that $\psi>0$. If $\alpha \in(\underline{\alpha}, \bar{\alpha})$, then

$$
h\left(f \mid K_{\alpha}\right)=\max \left\{h_{\mu}(f): \mu \in \mathcal{M}(X) \text { and } \frac{\int_{X} \varphi d \mu}{\int_{X} \psi d \mu}=\alpha\right\} .
$$

The statements in this section follow from more general statements formulated below (see Section [3.3). When $\psi=1$, the statement in Theorem 5 was established by Olivier [6] in the special case when $f$ is a full shift, with a substantially different approach. 
3.3. Generalizations. In this section we define precisely the most general situation for which we can establish a conditional variational principle.

The map $f: X \rightarrow X$ is now an arbitrary continuous map on the compact metric space $X$. We denote by $D(X) \subset C(X)$ the family of continuous functions with a unique equilibrium measure. Recall that a measure $\mu \in \mathcal{M}(X)$ is an equilibrium measure of a continuous function $\varphi: X \rightarrow \mathbb{R}$ if

$$
P_{X}(\varphi)=h_{\mu}(f)+\int_{X} \varphi d \mu,
$$

where $P_{X}(\varphi)$ denotes the topological pressure of $\varphi$ with respect to $f$ (see Section 2.1 for the definition).

Recall that if the metric entropy is upper semi-continuous, or, more precisely, if the map $\mu \mapsto h_{\mu}(f)$ is upper semi-continuous, then:

1. every function $\varphi \in C(X)$ has an equilibrium measure;

2. given $\varphi \in C(X)$, the function $q \mapsto P_{X}(\varphi+q \psi)$ is differentiable at $q=0$ for each $\psi \in C(X)$ if and only if $\varphi \in D(X)$; in this case the unique equilibrium measure $\mu_{\varphi}$ is ergodic, and

$$
\left.\frac{d}{d q} P_{X}(\varphi+q \psi)\right|_{q=0}=\int_{X} \psi d \mu_{\varphi} ;
$$

3. if $\varphi, \psi \in C(X)$ are such that $\operatorname{span}\{\varphi, \psi\} \subset D(X)$, then the function $q \mapsto$ $P_{X}(\varphi+q \psi)$ is differentiable on $\mathbb{R}$, and is in fact of class $C^{1}$ (see [5], Theorem 4.2.11]);

4. the family $D(X)$ is dense in $C(X)$ (see [8, Theorem 6.14]).

For example, when $f: X \rightarrow X$ is a one-sided or two-sided topologically mixing subshift of finite type, or an expansive homeomorphism, then the metric entropy is upper semi-continuous (see [5, Theorem 4.5.6]). Furthermore, if $f: X \rightarrow X$ is a one-sided or two-sided topologically mixing subshift of finite type, or an expansive homeomorphism which satisfies specification, and $\varphi \in C_{f}(X)$, then $\varphi$ has a unique equilibrium measure; see [4].

On the other hand, one can exhibit plenty transformations not satisfying specification for which the entropy is still upper semi-continuous. For example, all $\beta$-shifts are expansive, and thus the entropy is upper semi-continuous (see [5] for details), but for $\beta$ in a residual set of full Lebesgue measure (although the complement has full Hausdorff dimension) the corresponding $\beta$-shift does not satisfy specification (see [9]). It follows from work of Walters [12] that for every $\beta$-shift the set of Lipschitz functions is contained in $D(X)$. Theorem 6 below readily applies in this setting. However, the multifractal analysis of piecewise monotonic transformations is not yet completely understood.

The following theorem establishes a conditional variational principle for the multifractal spectrum $\mathcal{F}_{v}$.

Theorem 6. Assume that the metric entropy is upper semi-continuous, and let $\varphi$, $\psi, v \in C(X)$ with $\psi>0, v>0$, and such that $\operatorname{span}\{\varphi, \psi, v\} \subset D(X)$. Then the following properties hold:

1. if $\alpha \notin[\underline{\alpha}, \bar{\alpha}]$, then $K_{\alpha}=\emptyset$;

2. if $\alpha \in(\underline{\alpha}, \bar{\alpha})$, then $K_{\alpha} \neq \emptyset$ and

$$
\mathcal{F}_{v}(\alpha)=\max \left\{\frac{h_{\mu}(f)}{\int_{X} v d \mu}: \mu \in \mathcal{M}(X) \text { and } \frac{\int_{X} \varphi d \mu}{\int_{X} \psi d \mu}=\alpha\right\} ;
$$


3. the function $\mathcal{F}_{v}$ is continuous on $(\underline{\alpha}, \bar{\alpha})$.

Presumably, in general the function $\mathcal{F}_{v}$ need not be more regular than continuous. This contrasts with the situation in Section [5.1, where it is shown that if $f: X \rightarrow$ $X$ is a topologically mixing subshift of finite type, and $\varphi, \psi$, and $v$ are Hölder continuous functions, then $\mathcal{F}_{v}$ is even real analytic.

Taking $v=1$ in Theorem [ 6 one obtains a conditional variational principle for the topological entropy.

Theorem 7. Assume that the metric entropy is upper semi-continuous, and let $\varphi$, $\psi \in C(X)$ with $\psi>0$, and such that $\operatorname{span}\{\varphi, \psi\} \subset D(X)$. If $\alpha \in(\underline{\alpha}, \bar{\alpha})$, then

$$
h\left(f \mid K_{\alpha}\right)=\max \left\{h_{\mu}(f): \mu \in \mathcal{M}(X) \text { and } \frac{\int_{X} \varphi d \mu}{\int_{X} \psi d \mu}=\alpha\right\} .
$$

When $\psi=1$, the identity in Theorem 7 was established by Takens and Verbitski [1] under the assumption that $f$ is a continuous transformation satisfying specification, and $\varphi$ is an arbitrary continuous function (and thus may have more than one equilibrium measure), with a very different approach.

3.4. Relation with the topological pressure. We now show that the spectrum is given by an equation reminiscent of a Legendre transform of the topological pressure. However, we remark that the spectrum is in general not convex; see Proposition 10.

Theorem 8. Assume that the metric entropy is upper semi-continuous, and let $\varphi$, $\psi, v \in C(X)$ with $\psi>0, v>0$, and such that $\operatorname{span}\{\varphi, \psi, v\} \subset D(X)$. If $\alpha \in(\underline{\alpha}, \bar{\alpha})$ then:

1. $\inf _{q \in \mathbb{R}} P_{X}\left(q \varphi-q \alpha \psi-\mathcal{F}_{v}(\alpha) v\right)=0$;

2. if $\Delta(p, q)$ is the unique number such that $P_{X}(q \varphi-p \psi-\Delta(p, q) v)=0$, then

$$
\mathcal{F}_{v}(\alpha)=\inf _{q \in \mathbb{R}} \Delta(q \alpha, q) .
$$

Recall that two functions $\varphi$ and $\psi$ on $X$ are cohomologous (with respect to $f$ ) if there exists a continuous function $\chi: X \rightarrow \mathbb{R}$ such that

$$
\varphi-\psi=\chi \circ f-\chi \text {. }
$$

Under the hypotheses of Theorem 8 , assume, in addition, that $\psi$ is cohomologous to a multiple of $v$. One easily verifies that in this case $\psi$ must be cohomologous to $\lambda v$, where $\lambda$ is the unique number such that $P_{X}(\psi)=P_{X}(\lambda v)$. Thus

$$
P_{X}(q \varphi-[p \lambda+\Delta(p, q)] v)=0
$$

and hence, $T(q)=p \lambda+\Delta(p, q)$ does not depend on $p$. By (11), we obtain

$$
\mathcal{F}_{v}(\alpha)=\inf _{q \in \mathbb{R}}[T(q)-q \alpha \lambda] .
$$

We conclude that when $\psi$ is cohomologous to a multiple of $v$, the spectrum $\mathcal{F}_{v}$ is a Legendre transform, and hence it is convex. For example, this implies that for a conformal expanding map the "non-mixed" multifractal spectra $\mathcal{D}_{D}$ and $\mathcal{E}_{E}$ in Section 1 are always convex.

We also observe that under the hypotheses of Theorem 8 , if $v=1$ then

$$
h\left(f \mid K_{\alpha}\right)=\inf _{q \in \mathbb{R}} P_{X}(q \varphi-q \alpha \psi) .
$$




\section{Proofs of the Results in Section 3}

We start with some preparatory lemmas. In the sequel we shall always assume that the metric entropy is upper semi-continuous, and that $\varphi, \psi, v \in C(X)$ with $\psi>0$ and $v>0$, and such that $\operatorname{span}\{\varphi, \psi, v\} \subset D(X)$.

Lemma 1. If $\alpha \in \mathbb{R}$, then

$$
\inf _{q \in \mathbb{R}} P_{X}\left(q \varphi-q \alpha \psi-\mathcal{F}_{v}(\alpha) v\right) \geq 0 .
$$

Proof of the lemma. By Proposition 2 the number $\mathcal{F}_{v}(\alpha)$ coincides with the unique root $\delta$ of the equation $P_{K_{\alpha}}(-\delta v)=0$. Given $\delta>0$ and $\tau \in \mathbb{N}$ consider the sets

$$
L_{\delta, \tau}=\left\{x \in X:\left|\varphi_{n}(x)-\alpha \psi_{n}(x)\right|<\delta n \text { for every } n \geq \tau\right\},
$$

where

$$
\varphi_{n}=\sum_{k=0}^{n-1} \varphi \circ f^{k} \quad \text { and } \quad \psi_{n}=\sum_{k=0}^{n-1} \psi \circ f^{k} .
$$

Since $\psi>0$ one can easily show that $K_{\alpha} \subset \bigcap_{\delta>0} \bigcup_{\tau \in \mathbb{N}} L_{\delta, \tau}$. Let now $\mathcal{U}$ be an open cover of $X$ with sufficiently small diameter such that if $n$ is sufficiently large, $\mathbf{U} \in \bigcup_{k \geq n} \mathcal{W}_{k}(\mathcal{U})$, and $x \in X(\mathbf{U})$, then

$$
\left|\varphi(\mathbf{U})-\varphi_{m(\mathbf{U})}(x)\right| \leq \delta m(\mathbf{U}) \text { and }\left|\psi(\mathbf{U})-\psi_{m(\mathbf{U})}(x)\right| \leq \delta m(\mathbf{U}) .
$$

Hence, if $\mathbf{U} \in \bigcup_{k \geq n} \mathcal{W}_{k}(\mathcal{U})$ and $X(\mathbf{U}) \cap L_{\delta, \tau} \neq \emptyset$, then

$$
|\varphi(\mathbf{U})-\alpha \psi(\mathbf{U})|<(2+|\alpha|) \delta m(\mathbf{U}) .
$$

Thus

$$
P_{L_{\delta, \tau}}\left(-\mathcal{F}_{v}(\alpha) v, \mathcal{U}\right) \leq P_{L_{\delta, \tau}}\left(q \varphi-q \alpha \psi-\mathcal{F}_{v}(\alpha) v, \mathcal{U}\right)+(2+|\alpha|) \delta|q| .
$$

Letting the diameter of $\mathcal{U}$ go to zero yields

$$
P_{L_{\delta, \tau}}\left(-\mathcal{F}_{v}(\alpha) v\right) \leq P_{X}\left(q \varphi-q \alpha \psi-\mathcal{F}_{v}(\alpha) v\right)+(2+|\alpha|) \delta|q|,
$$

and hence,

$$
\begin{aligned}
0 & \leq P_{\cup_{\tau \in \mathbb{N}} L_{\delta, \tau}}\left(-\mathcal{F}_{v}(\alpha) v\right)=\sup _{\tau \in \mathbb{N}} P_{L_{\delta, \tau}}\left(-\mathcal{F}_{v}(\alpha) v\right) \\
& \leq P_{X}\left(q \varphi-q \alpha \psi-\mathcal{F}_{v}(\alpha) v\right)+(2+|\alpha|) \delta|q| .
\end{aligned}
$$

Since $\delta$ is arbitrary, we obtain

$$
\inf _{q \in \mathbb{R}} P_{X}\left(q \varphi-q \alpha \psi-\mathcal{F}_{v}(\alpha) v\right) \geq 0 .
$$

This completes the proof.

In the sequel we shall always denote by $\zeta=\zeta_{q, \alpha, \delta}$ the unique equilibrium measure of $q \varphi-q \alpha \psi-\delta v$, which is well-defined in our setting (see Section 3.3).

Lemma 2. For each $\alpha \in(\underline{\alpha}, \bar{\alpha})$ and $\delta \in \mathbb{R}$ there exists $q=q(\delta, \alpha)$ such that $\int_{X} \varphi d \zeta / \int_{X} \psi d \zeta=\alpha$

Proof of the lemma. Let $\delta \in \mathbb{R}$ and $\alpha \in(\underline{\alpha}, \bar{\alpha})$. Define a function $S: \mathbb{R} \rightarrow \mathbb{R}$ by

$$
S(q) \stackrel{\text { def }}{=} \int_{X} \varphi d \zeta-\alpha \int_{X} \psi d \zeta=\frac{d}{d q} P_{X}(q \varphi-q \alpha \psi-\delta v) .
$$

It follows from the upper semi-continuity of the entropy that the function $q \mapsto$ $P_{X}(q \varphi-q \alpha \psi-\delta v)$ is of class $C^{1}$ (see for example [5, Theorem 4.2.11]), and thus $S$ is continuous. 
We shall prove that $S(q)>0$ if $q>0$ is sufficiently large. Since $\zeta$ is an equilibrium measure, if $q>0$ then

$$
\begin{aligned}
S(q)= & \frac{1}{q}\left[P_{X}(q \varphi-q \alpha \psi-\delta v)+\delta \int_{X} v d \zeta-h_{\zeta}(f)\right] \\
= & \sup _{\mu}\left[\int_{X} \varphi d \mu-\alpha \int_{X} \psi d \mu+\frac{\delta\left(\int_{X} v d \zeta-\int_{X} v d \mu\right)+h_{\mu}(f)-h_{\zeta}(f)}{q}\right] \\
\geq & \sup _{\mu}\left[\int_{X} \varphi d \mu-\bar{\alpha} \int_{X} \psi d \mu+\frac{\delta\left(\int_{X} v d \zeta-\int_{X} v d \mu\right)+h_{\mu}(f)-h_{\zeta}(f)}{q}\right] \\
& +(\bar{\alpha}-\alpha) \inf \psi .
\end{aligned}
$$

Since $v$ and the entropies are bounded and $(\bar{\alpha}-\alpha) \inf \psi>0$, we conclude that $S(q)>0$ for all sufficiently large $q>0$. A similar argument shows that $S(q)<0$ for all sufficiently small $q<0$. The desired result follows from the continuity of $S$.

Lemma 3. Given $\alpha \in(\underline{\alpha}, \bar{\alpha})$, if $\delta$ is such that $P_{X}(q \varphi-q \alpha \psi-\delta v) \geq 0$ for every $q \in \mathbb{R}$, then there exists an ergodic measure $\mu \in \mathcal{M}(X)$ such that

$$
\int_{X} \varphi d \mu / \int_{X} \psi d \mu=\alpha \quad \text { and } \quad \operatorname{dim}_{v} \mu \geq \delta
$$

Proof of the lemma. Let $q=q(\delta, \alpha)$ be as in Lemma 2 and continue to write $\zeta=\zeta_{q, \alpha, \delta}$. Since $P_{X}(q \varphi-q \alpha \psi-\delta v) \geq 0$ we obtain

$$
h_{\zeta}(f)+q \int_{X} \varphi d \zeta-q \alpha \int_{X} \psi d \zeta-\delta \int_{X} v d \zeta \geq 0 .
$$

By Proposition 3, we conclude that $\delta \leq h_{\zeta}(f) / \int_{X} v d \zeta=\operatorname{dim}_{v} \zeta$.

Lemma 4. If $\alpha \in(\underline{\alpha}, \bar{\alpha})$, then $K_{\alpha} \neq \emptyset$ and

$$
\mathcal{F}_{v}(\alpha)=\sup \left\{\operatorname{dim}_{v} \mu: \mu \in \mathcal{M}_{E}(X) \text { and } \frac{\int_{X} \varphi d \mu}{\int_{X} \psi d \mu}=\alpha\right\} .
$$

Proof of the lemma. By Lemma 1 we can apply Lemma 3 with $\delta=\mathcal{F}_{v}(\alpha)$ to obtain

$$
\mathcal{F}_{v}(\alpha) \leq \sup \left\{\operatorname{dim}_{v} \mu: \mu \in \mathcal{M}_{E}(X) \text { and } \frac{\int_{X} \varphi d \mu}{\int_{X} \psi d \mu}=\alpha\right\} .
$$

Let $\mu \in \mathcal{M}_{E}(X)$ be a measure such that

$$
\int_{X} \varphi d \mu / \int_{X} \psi d \mu=\alpha
$$

Birkhoff's ergodic theorem implies that $\mu\left(K_{\alpha}\right)=1$, and hence, by Proposition 3 we obtain

$$
\mathcal{F}_{v}(\alpha) \geq \operatorname{dim}_{v} \mu=\frac{h_{\mu}(f)}{\int_{X} v d \mu}
$$

This completes the proof.

Lemma 5. If $\alpha \in(\underline{\alpha}, \bar{\alpha})$ then

$$
\inf _{q \in \mathbb{R}} P_{X}\left(q \varphi-q \alpha \psi-\mathcal{F}_{v}(\alpha) v\right)=0 .
$$


Proof of the lemma. Since $v$ is positive it follows from Lemma 1 and the continuity of the topological pressure (in the supremum norm) that there exists $\delta^{*} \geq \mathcal{F}_{v}(\alpha)$ such that

$$
\inf _{q \in \mathbb{R}} P_{X}\left(q \varphi-q \alpha \psi-\delta^{*} v\right)=0 .
$$

On the other hand, Lemma 3 ensures the existence of an ergodic measure $\mu$ such that

$$
\int_{X} \varphi d \mu / \int_{X} \psi d \mu=\alpha \text { and } \operatorname{dim}_{v} \mu \geq \delta^{*}
$$

Lemma 4 yields $\mathcal{F}_{v}(\alpha) \geq \delta^{*}$. This completes the proof.

We now establish the continuity of the spectrum.

Lemma 6. The function $\mathcal{F}_{v}$ is continuous on $(\underline{\alpha}, \bar{\alpha})$.

Proof of the lemma. We first show that $\mathcal{F}_{v}$ is upper semi-continuous. Let $\alpha \in(\underline{\alpha}, \bar{\alpha})$ and $\alpha_{n} \in(\underline{\alpha}, \bar{\alpha})$ be any sequence converging to $\alpha$. By Lemma 2 there exists $q_{n} \in \mathbb{R}$ such that

$$
\int_{X} \varphi d \mu_{n}=\alpha_{n} \int_{X} \psi d \mu_{n}
$$

where $\mu_{n}=\zeta_{q_{n}, \alpha_{n}, \mathcal{F}_{v}\left(\alpha_{n}\right)}$. By Lemma 5 the function

$$
q \mapsto P_{X}\left(q \varphi-q \alpha_{n} \psi-\mathcal{F}_{v}\left(\alpha_{n}\right) v\right)
$$

attains its infimum (which is zero) at $q=q_{n}$. Since $\mu_{n}$ is an equilibrium measure, we have

$$
h_{\mu_{n}}(f)=\mathcal{F}_{v}\left(\alpha_{n}\right) \int_{X} v d \mu_{n}
$$

Let $\beta=\lim _{\sup _{n \rightarrow \infty}} \mathcal{F}_{v}\left(\alpha_{n}\right)$. Taking a subsequence, if necessary, we may assume that $\mathcal{F}_{v}\left(\alpha_{n}\right)$ converges to $\beta$ and that the sequence of measures $\mu_{n}$ converges weakly to some measure $\mu$. Since the entropy is upper semi-continuous, we obtain

$$
h_{\mu}(f) \geq \limsup _{n \rightarrow \infty} h_{\mu_{n}}(f)=\limsup _{n \rightarrow \infty} \mathcal{F}_{v}\left(\alpha_{n}\right) \int_{X} v d \mu .
$$

Since $\mu_{n} \rightarrow \mu$ and $\alpha_{n} \rightarrow \alpha$, we have $\int_{X} \varphi d \mu-\alpha \int_{X} \psi d \mu=0$, and hence if $q \in \mathbb{R}$ then

$$
P_{X}\left(q \varphi-q \alpha \psi-\mathcal{F}_{v}(\alpha) v\right) \geq h_{\mu}(f)-\mathcal{F}_{v}(\alpha) \int_{X} v d \mu .
$$

Taking the infimum over $q$, by Lemma 5 we obtain $\mathcal{F}_{v}(\alpha) \int_{X} v d \mu \geq h_{\mu}(f)$. Since $v>0$, by (14) we conclude that $\mathcal{F}_{v}$ is upper semi-continuous.

We now show that $\mathcal{F}_{v}$ is lower semi-continuous. Let $\alpha_{*} \in(\underline{\alpha}, \bar{\alpha})$. We define

$$
\chi_{q}=q \varphi-q \alpha_{*} \psi-\mathcal{F}_{v}\left(\alpha_{*}\right) v \text { and } F(q)=P_{X}\left(\chi_{q}\right) .
$$

By Lemma 1 we have $F(q) \geq 0$ for every $q$. The function $F$ is of class $C^{1}$ and its derivative is

$$
S(q)=\int_{X} \varphi d \mu_{q}-\alpha_{*} \int_{X} \psi d \mu_{q}
$$

where $\mu_{q}$ denotes the equilibrium measure of $\chi_{q}$. We note that $S$ is increasing, by the convexity of the topological pressure. By Lemma 2 there exists $q_{*}=q_{*}\left(\alpha_{*}\right) \in \mathbb{R}$ such that $S\left(q_{*}\right)=0$. It is shown in the proof of Lemma 2 that $S(q)>0$ if $q>0$ is 
sufficiently large, and that $S(q)<0$ if $q<0$ is sufficiently small. Therefore, we can always choose $q_{*}$ in such a way that $S(q)>0$ for every $q>q_{*}$ (in what follows it is in fact enough that any neighborhood of $q_{*}$ contains points where $S$ is positive).

Let $\varepsilon>0$. Since $S$ is continuous, one can always choose $\delta \in(0, \varepsilon)$ such that

$$
\sup \left\{|S(q)|: q \in\left(q_{*}-\delta, q_{*}+\delta\right)\right\} \leq \varepsilon \inf v .
$$

Since $\mu_{q}$ is the equilibrium measure of $\chi_{q}$ and $F(q) \geq 0$, it follows from Proposition3 that

$$
\begin{aligned}
\operatorname{dim}_{v} \mu_{q}=\frac{h_{\mu_{q}}(f)}{\int_{X} v d \mu_{q}} & =\mathcal{F}_{v}\left(\alpha_{*}\right)+\frac{F(q)-q S(q)}{\int_{X} v d \mu_{q}} \\
& \geq \mathcal{F}_{v}\left(\alpha_{*}\right)-\frac{q S(q)}{\int_{X} v d \mu_{q}} \geq \mathcal{F}_{v}\left(\alpha_{*}\right)-\left(\left|q_{*}\right|+\varepsilon\right) \varepsilon .
\end{aligned}
$$

For every $\alpha>\alpha_{*}$ sufficiently small it follows from the continuity of $S$ and the choice of $q_{*}$ that there exists $q=q(\alpha) \in\left(q_{*}, q_{*}+\delta\right)$ such that $\alpha=\alpha_{*}+S(q) / \int_{X} \psi d \mu_{q}$, and hence $\int_{X} \varphi d \mu_{q}=\alpha \int_{X} \psi d \mu_{q}$. By Lemma 4 and (15) we conclude that

$$
\mathcal{F}_{v}(\alpha) \geq \operatorname{dim}_{v} \mu_{q} \geq \mathcal{F}_{v}\left(\alpha_{*}\right)-\left(\left|q_{*}\right|+\varepsilon\right) \varepsilon .
$$

Since $\varepsilon$ is arbitrary, this establishes the right lower semi-continuity of $\mathcal{F}_{v}$.

The left lower semi-continuity can be established in a similar way. If necessary we first rechoose $q_{*}$ in such a way that $S(q)<0$ for every $q<q_{*}$. For every $\alpha<\alpha_{*}$ sufficiently small there exists $q=q(\alpha) \in\left(q_{*}-\delta, q_{*}\right)$ such that $\alpha=\alpha_{*}+$

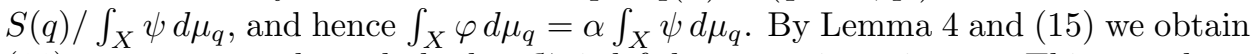
(16) once more, and conclude that $\mathcal{F}_{v}$ is left lower semi-continuous. This completes the proof of the lemma.

We now establish the results in Section 3

Proof of Theorem [6] Setting $\delta=\mathcal{F}_{v}(\alpha)$ in Lemma 2 we conclude that the ergodic measure $\zeta=\zeta_{q, \alpha, \delta}$ satisfies

$$
P_{X}\left(q \varphi-q \alpha \psi-\mathcal{F}_{v}(\alpha) v\right)=h_{\zeta}(f)-\mathcal{F}_{v}(\alpha) \int_{X} v d \zeta
$$

and hence, $\mathcal{F}_{v}(\alpha)=h_{\zeta}(f) / \int_{X} v d \zeta=\operatorname{dim}_{v} \zeta$. This shows that in Lemma4 4 one can replace each of the suprema by a maximum.

Let now $\mu \in \mathcal{N}(X)$ be a measure such that $\int_{X} \varphi d \mu / \int_{X} \psi d \mu=\alpha$ (note that $\mu$ is not necessarily ergodic). To complete the proof of Statement 2 it is enough to show that $\mathcal{F}_{v}(\alpha) \geq h_{\mu}(f) / \int_{X} v d \mu$. Observe that

$$
\begin{aligned}
& P_{X}\left(q \varphi-q \alpha \psi-\mathcal{F}_{v}(\alpha) v\right) \\
& \geq h_{\mu}(f)+q \int_{X} \varphi d \mu-q \alpha \int_{X} \psi d \mu-\mathcal{F}_{v}(\alpha) \int_{X} v d \mu \\
& =h_{\mu}(f)-\mathcal{F}_{v}(\alpha) \int_{X} v d \mu .
\end{aligned}
$$

Taking the infimum over $q$, Lemma 5 yields $0 \geq h_{\mu}(f)-\mathcal{F}_{v}(\alpha) \int_{X} v d \mu$, and hence,

$$
\mathcal{F}_{v}(\alpha) \geq \frac{h_{\mu}(f)}{\int_{X} v d \mu}
$$

as we wanted to show. This establishes Statement 2. Statement 1 is an immediate consequence of Statement 2. 
Finally, the continuity of $\mathcal{F}_{v}$ is established in Lemma [6. This completes the proof.

Proof of Theorem 4. It is well known that for topologically mixing subshifts of finite type and potentials in $C_{f}(X)$ the assumptions in Theorem 6 are satisfied. The desired statement is thus an immediate consequence of Theorem 6

Proof of Theorem [8. Statement 1 is established in Lemma 5. Statement 2 is an immediate consequence of Statement 1.

\section{Applications}

5.1. Analyticity and non-convexity of the spectrum. We continue to use the notations of Section [3. We show that for subshifts of finite type the multifractal spectrum $\mathcal{F}_{v}$ is real analytic, thus substantially improving Statement 3 in Theorem 6 for this class of dynamical systems.

Theorem 9. Let $f: X \rightarrow X$ be a topologically mixing subshift of finite type, and $\varphi, \psi$, and $v$ Hölder continuous functions on $X$ with $\psi>0$ and $v>0$. Then the following properties hold:

1. If $\varphi$ is cohomologous to some multiple of $\psi$, then $\underline{\alpha}=\bar{\alpha}$.

2. If $\varphi$ is not cohomologous to any multiple of $\psi$, then the function $\mathcal{F}_{v}$ is real analytic on the non-empty interval $(\underline{\alpha}, \bar{\alpha})$.

Proof. Assume first that there exists $\beta \in \mathbb{R}$ and a continuous function $\chi: X \rightarrow \mathbb{R}$ such that $\varphi-\beta \psi=\chi \circ f-\chi$. Then

$$
\frac{\varphi_{n}}{\psi_{n}}-\beta=\frac{\chi \circ f^{n}-\chi}{\psi_{n}} \rightarrow 0 \text { as } n \rightarrow \infty .
$$

Hence, $K_{\beta}(\varphi, \psi)=X$ and $K_{a}(\varphi, \psi)=\emptyset$ for every $a \neq \beta$. By Birkhoff's ergodic theorem, if $\mu \in \mathcal{M}(X)$ is ergodic then $\int_{X} \varphi d \mu / \int_{X} \psi d \mu=\beta$, and hence, $\underline{\alpha}=\bar{\alpha}=\beta$. This establishes the first property.

We now prove an auxiliary lemma.

Lemma 7. If $\varphi-\beta \psi$ is cohomologous to no constant for every $\beta \in \mathbb{R}$, then the spectrum $\mathcal{F}_{v}$ is real analytic on the non-empty interval $(\underline{\alpha}, \bar{\alpha})$.

Proof of the lemma. Let $\alpha \in(\underline{\alpha}, \bar{\alpha})$ and put

$$
F(q, \delta, \alpha)=P_{X}(q \varphi-q \alpha \psi-\delta v) .
$$

By Theorem 8 the number $\mathcal{F}_{v}(\alpha)$ coincides with the unique $\delta$ such that $\inf _{q} F(q, \delta, \alpha)$ $=0$. It is well known that $F$ is real analytic in all variables. In addition, the function $q \mapsto F(q, \delta, \alpha)$ is strictly convex because of the cohomology assumption. Hence if there exists $q \in \mathbb{R}$ such that its derivative $\partial_{q} F$ vanishes, then this is the minimum. But

$$
\partial_{q} F(q, \delta, \alpha)=S(q)
$$

with $S(q)$ as in (13). It is shown in the proof of Lemma 2 that there exists a point $q=q(\delta, \alpha) \in \mathbb{R}$ such that $S(q)=0$; such a point is necessarily unique by the strict convexity of $q \mapsto F(q, \delta, \alpha)$. The dimension is then given by the unique root $\delta=\delta(\alpha)$ of the system of equations

$$
F(q(\delta, \alpha), \delta, \alpha)=\partial_{q} F(q(\delta, \alpha), \delta, \alpha)=0 .
$$


We want to apply the implicit function theorem in order to get the regularity of $q(\alpha)=q(\delta(\alpha), \alpha)$ and $\delta(\alpha)$. To do this, let

$$
G(q, \delta, \alpha)=\left(\begin{array}{c}
F(q, \delta, \alpha) \\
\partial_{q} F(q, \delta, \alpha)
\end{array}\right)
$$

It is enough to show that

$$
\operatorname{det}\left[\left(\partial_{q}, \partial_{\delta}\right) G\right]=\partial_{q} F \cdot \partial_{\delta} \partial_{q} F-\partial_{q}^{2} F \cdot \partial_{\delta} F
$$

never vanishes when $\delta=\delta(\alpha)$ and $q=q(\delta(\alpha), \alpha)$. Since $\partial_{q} F=0$ on $q(\delta, \alpha)$, it is enough to check that $\partial_{q}^{2} F$ and $\partial_{\delta} F$ are non-zero. Observe that $\int_{X}(\varphi-\alpha \psi) d_{\zeta_{q, \alpha, \delta}}=$ 0 when $q=q(\delta(\alpha), \alpha)$ and $\delta=\delta(\alpha)$. Since $\varphi-\alpha \psi$ is not cohomologous to a constant $\partial_{q}^{2} F$ does not vanish (see [8]). Finally,

$$
\partial_{\delta} F=-\int_{X} v d \zeta_{q, \alpha, \delta} \leq-\inf v<0 .
$$

This proves that $\delta(\alpha)$ and $q(\alpha)$ are analytic.

We proceed with the proof of the theorem. Assume that $\varphi$ is not cohomologous to $\beta \psi$ for every $\beta \in \mathbb{R}$. We want to prove that $\underline{\alpha}<\bar{\alpha}$, and that the spectrum $\mathcal{F}_{v}$ is real analytic on $(\underline{\alpha}, \bar{\alpha})$.

By Lemma 7 , it remains to consider the case when there exists $\beta, c \in \mathbb{R}$ with $c \neq 0$, and a continuous function $\chi: X \rightarrow \mathbb{R}$ such that

$$
\varphi-\beta \psi=c+\chi \circ f-\chi
$$

One can easily show that $x \in K_{\alpha}(\varphi, \psi)$ if and only if $x \in K_{c /(\alpha-\beta)}(\psi, 1)$. Furthermore, it follows from (17) that

$$
\frac{\varphi_{n}}{\psi_{n}}-\beta=\frac{c n}{\psi_{n}}+\frac{\chi \circ f^{n}-\chi}{\psi_{n}} .
$$

Since $\psi>0$ and $c \neq 0$ we conclude that $\beta \neq \alpha$ for every $\alpha \in \mathbb{R}$ such that $K_{\alpha}(\varphi, \psi) \neq \emptyset$. Hence, the function $\alpha \mapsto c /(\alpha-\beta)$ is real analytic on $(\underline{\alpha}, \bar{\alpha})$.

Observe that $\psi$ cannot be cohomologous to a constant $\gamma \in \mathbb{R}$. Otherwise the function $\varphi$ would be cohomologous to $\beta \psi+c=(\beta+c / \gamma) \psi$ (since $\psi>0$ the constant $\gamma$ would be positive), which is a contradiction. Hence we can apply Lemma 7 to the pair of functions $(\psi, 1)$ to conclude that $\mathcal{F}_{v}^{(\psi, 1)}$ is real analytic on the non-empty interval $(\underline{\beta}, \bar{\beta})$, where

$$
\begin{aligned}
& \underline{\beta}=\inf \left\{\int_{X} \psi d \mu: \mu \in \mathcal{M}(X)\right\}, \\
& \bar{\beta}=\sup \left\{\int_{X} \psi d \mu: \mu \in \mathcal{M}(X)\right\} .
\end{aligned}
$$

Since $\psi>0$ we have $\underline{\beta}>0$.

Since $\mathcal{F}_{v}^{(\varphi, \psi)}$ is the composition of the real analytic functions $\alpha \mapsto c /(\alpha-\beta)$ and $\mathcal{F}_{v}^{(\psi, 1)}$, we conclude that it is real analytic. Furthermore,

$$
(\underline{\alpha}, \bar{\alpha})= \begin{cases}(\beta+c / \bar{\beta}, \beta+c / \underline{\beta}) & \text { when } c>0, \\ (\beta+c / \underline{\beta}, \beta+c / \overline{\bar{\beta}}) & \text { when } c<0 .\end{cases}
$$

This completes the proof of the theorem. 


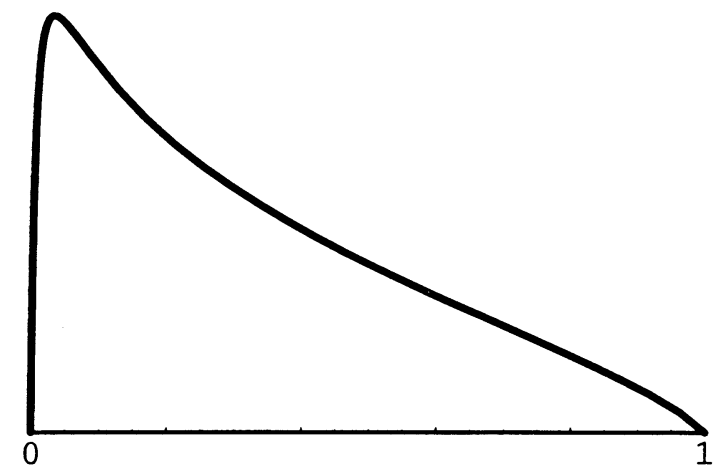

Figure 1. A non-convex spectrum

We now provide an explicit example for which the spectrum $\mathcal{F}_{v}$ is not convex. This strongly contrasts with the well studied (non-mixed) multifractal spectra, which can be computed as Legendre transforms, and hence are always convex.

Proposition 10. Let $f: X \rightarrow X$ be the full shift on two symbols. There exist Hölder continuous functions $\varphi, \psi$, and $v$ on $X$, depending only on the first coordinates, such that the spectrum $\mathcal{F}_{v}$ is not convex, and hence it is not a Legendre transform.

Construction. Let $X=\{0,1\}^{\mathbb{N}}$. Set $\varphi\left(x_{0} x_{1} \ldots\right)=\varphi_{x_{0}}$, with $\varphi_{0}=0$ and $\varphi_{1}=1$, $\psi=1$, and $v\left(x_{0} x_{1} \ldots\right)=v_{x_{0}}$ for some positive numbers $v_{0}$ and $v_{1}$. Write $\beta_{\mu}=$ $\mu\left(\left\{x \in X: x_{0}=1\right\}\right)$. By Theorem 4 , we have

$$
\mathcal{F}_{v}(\alpha)=\max \left\{\frac{h_{\mu}(f)}{v_{0}\left(1-\beta_{\mu}\right)+v_{1} \beta_{\mu}}: \beta_{\mu}=\alpha\right\}=\frac{\max \left\{h_{\mu}(f): \beta_{\mu}=\alpha\right\}}{-\alpha\left(v_{0}-v_{1}\right)+v_{0}} .
$$

Let $\xi$ be the partition of $X$ into cylinders of length 1 . Then

$$
h_{\mu}(f)=\inf _{n} \frac{1}{n} H_{\mu}\left(\bigvee_{k=0}^{n-1} f^{-k} \xi\right) \leq H_{\mu}(\xi)=-\alpha \log \alpha-(1-\alpha) \log (1-\alpha),
$$

with equality if $\mu$ is the Bernoulli measure on $\xi$ with $\beta_{\mu}=\alpha$. Therefore

$$
\mathcal{F}_{v}(\alpha)=\frac{\alpha \log \alpha+(1-\alpha) \log (1-\alpha)}{\alpha\left(v_{0}-v_{1}\right)-v_{0}} .
$$

Taking, for example, $v_{0}=1$ and $v_{1}=100$, the function $\mathcal{F}_{v}$ is not convex. See Figure 1 for a graph of the spectrum $\mathcal{F}_{v}$.

5.2. Irregular sets. Consider functions $\varphi, \psi \in C(X)$ such that $\psi>0$. Note that the space $X$ can be written as the disjoint union

$$
X=\bigcup_{\alpha \in[\underline{\alpha}, \bar{\alpha}]} K_{\alpha}(\varphi, \psi) \cup I(\varphi, \psi),
$$

where

$$
I(\varphi, \psi)=\left\{x \in X: \lim _{n \rightarrow \infty} \frac{\varphi_{n}(x)}{\psi_{n}(x)} \text { does not exist }\right\}
$$


is the irregular set for the pair $(\varphi, \psi)$. By Birkhoff's ergodic theorem the set $I(\varphi, \psi)$ has zero measure with respect to any invariant measure. The following result shows that from the point of view of dimension theory the set $I(\varphi, \psi)$ is as large as the whole set $X$.

We recall that a subshift is said to satisfy the specification property if there exists a positive integer $m$ such that for all cylinders $C_{1}, C_{2}$ there exists another cylinder $C$ of length $m$ such that the juxtaposition $C_{1} C C_{2}$ is a cylinder appearing in the subshift.

Theorem 11. Let $f: X \rightarrow X$ be a subshift with the specification property, and $\varphi$, $\psi, v \in C(X)$ with $\psi>0$ and $v>0$. If $\operatorname{span}\{\varphi, \psi, v\} \subset D(X)$ and $\underline{\alpha}<\bar{\alpha}$, then

$$
\operatorname{dim}_{v} I(\varphi, \psi)=\operatorname{dim}_{v} X .
$$

Proof. By Theorem [6 the function $\mathcal{F}_{v}$ is continuous on $(\underline{\alpha}, \bar{\alpha})$. We want to argue that the function $\mathcal{F}_{v}$ is also continuous where it attains its maximum even if it occurs when $\alpha \in\{\underline{\alpha}, \bar{\alpha}\}$. Let $\mu_{v}$ be the equilibrium measure of the potential

$$
-\operatorname{dim}_{v} X \cdot v=0(\varphi-\alpha \psi)-\operatorname{dim}_{v} X \cdot v .
$$

Since $\mu_{v}$ is the unique $f$-invariant probability measure such that $\operatorname{dim}_{v} \mu_{v}=\operatorname{dim}_{v} X$, we have $\mathcal{F}_{v}(\alpha) \leq \operatorname{dim}_{v} \mu_{v}$ for every $\alpha$. One can easily see that if

$$
\alpha=\int_{X} \varphi d \mu_{v} / \int_{X} \psi d \mu_{v}
$$

then $\mathcal{F}_{v}(\alpha)=\operatorname{dim}_{v} \mu_{v}$. Thus, if $\alpha \in\{\underline{\alpha}, \bar{\alpha}\}$ then one can use a argument similar to that in the proof of Lemma 6 to establish the continuity at $\alpha$.

We need the following statement, which is an immediate consequence of a result of Barreira and Schmeling (see [3] Theorem 7.2]).

Lemma 8. If $f: X \rightarrow X$ is a subshift with the specification property, and $\mu_{1}$, $\mu_{2} \in \mathcal{M}_{E}(X)$ are such that

$$
\frac{\int_{X} \varphi d \mu_{1}}{\int_{X} \psi d \mu_{1}} \neq \frac{\int_{X} \varphi d \mu_{2}}{\int_{X} \psi d \mu_{2}}
$$

then $\operatorname{dim}_{v} I(\varphi, \psi) \geq \min \left\{\operatorname{dim}_{v} \mu_{1}, \operatorname{dim}_{v} \mu_{2}\right\}$.

The continuity of $\mathcal{F}_{v}$ at the point $\alpha=\int_{X} \varphi d \mu_{v} / \int_{X} \psi d \mu_{v}$ assures that for each $\varepsilon>0$ there exists a measure $\mu \in \mathcal{M}_{E}(X)$ (which is an equilibrium measure) such that $\int_{X} \varphi d \mu / \int_{X} \psi d \mu \neq \alpha$ and $\operatorname{dim}_{v} \mu>\operatorname{dim}_{v} X-\varepsilon$. By Lemma 8 we conclude that

$$
\operatorname{dim}_{v} I(\varphi, \psi) \geq \min \left\{\operatorname{dim}_{v} \mu_{v}, \operatorname{dim}_{v} \mu\right\}>\operatorname{dim}_{v} X-\varepsilon .
$$

The arbitrariness of $\varepsilon$ implies the desired result.

For example, if the continuous functions $\varphi, \psi$, and $v$ are in $C_{f}(X)$, then $\operatorname{span}\{\varphi, \psi, v\} \subset D(X)$. In the special case of topologically mixing subshifts of finite type and Hölder continuous functions, the statement in Theorem[11 is due to Barreira and Schmeling [3]. The proof of Theorem[1] also uses their techniques.

When $f: X \rightarrow X$ is a subshift with the specification property, and $\varphi_{i}, \psi_{i}$, $v \in C(X)$ with $\psi_{i}>0$ and $v>0$, are such that $\operatorname{span}\left\{\varphi_{i}, \psi_{i}, v\right\} \subset D(X)$ and 
$\underline{\alpha}\left(\varphi_{i}, \psi_{i}\right)<\bar{\alpha}\left(\varphi_{i}, \psi_{i}\right)$ for $i=1, \ldots, k$, following the techniques in 3$]$ one can also show that

$$
\operatorname{dim}_{v} \bigcap_{i=1}^{k} I\left(\varphi_{i}, \psi_{i}\right)=\operatorname{dim}_{v} X
$$

For topologically mixing subshifts of finite type, this statement was established in [3].

5.3. Repellers. Let $M$ be a smooth Riemannian manifold and $f: M \rightarrow M$ a $C^{1}$ map. Consider a compact $f$-invariant set $X \subset M$. We say that $f$ is expanding and that $X$ is a repeller of $f$ if there exist constants $c>0$ and $\beta>1$ such that $\left\|d_{x} f^{n} u\right\| \geq c \beta^{n}\|u\|$ for all $x \in X, u \in T_{x} M$, and $n \geq 1$. if:

Recall that a finite cover $\left\{R_{1}, \ldots, R_{p}\right\}$ of $X$ by closed sets is a Markov partition

1. $\overline{\operatorname{int} R_{i}}=R_{i}$ for each $i=1, \ldots, p$;

2. int $R_{i} \cap \operatorname{int} R_{j}=\emptyset$ if $i \neq j$;

3. each $f R_{i}$ is a union of sets $R_{j}$.

It is well known that repellers admit Markov partitions of arbitrarily small diameter. We define a $p \times p$ transfer matrix $A=\left(a_{i j}\right)$ by setting $a_{i j}=1$ if int $R_{i} \cap f^{-1}$ int $R_{j} \neq$ $\emptyset$, and $a_{i j}=0$ otherwise. Consider the associated subshift of finite type $\sigma: \Sigma \rightarrow \Sigma$. For each $\omega=\left(i_{1} i_{2} \cdots\right) \in \Sigma$, we set

$$
\chi(\omega)=\left\{x \in X: f^{k-1} x \in R_{i_{k}} \text { for every } k \in \mathbb{N}\right\} .
$$

The set $\chi(\omega)$ consists of a single point in $X$, and we obtain a coding map $\chi: \Sigma \rightarrow X$. The map $\chi$ is continuous, onto, and satisfies $\chi \circ \sigma=f \circ \chi$.

For expanding maps, one can also establish a conditional variational principle for the $v$-dimension, and the analyticity of the spectrum $\mathcal{F}_{v}$. Furthermore, one can establish the full $v$-dimensionality of irregular sets, and relate the spectrum with a Legendre-type transform. We collect all the results in the following statement.

Theorem 12. Let $X$ be a repeller of a topologically mixing $C^{1+\varepsilon}$ expanding map $f$, for some $\varepsilon>0$, and $\varphi, \psi, v$ Hölder continuous on $X$ with $\psi>0$ and $v>0$. If $\varphi$ is not cohomologous to any multiple of $\psi$, then:

1. the function $\mathcal{F}_{v}$ is real analytic on $(\underline{\alpha}, \bar{\alpha})$;

2. if $\alpha \in(\underline{\alpha}, \bar{\alpha})$ then

$$
\mathcal{F}_{v}(\alpha)=\max \left\{\frac{h_{\mu}(f)}{\int_{X} v d \mu}: \mu \in \mathcal{M}(X) \text { and } \frac{\int_{X} \varphi d \mu}{\int_{X} \psi d \mu}=\alpha\right\}=\inf _{q \in \mathbb{R}} \Delta(q \alpha, q) \text {, }
$$

where $\Delta(p, q)$ is such that $P_{X}(q \varphi-p \psi-\Delta(p, q) v)=0$;

3. $\inf _{q \in \mathbb{R}} P_{X}\left(q \varphi-q \alpha \psi-\mathcal{F}_{v}(\alpha) v\right)=0$ for every $\alpha \in(\underline{\alpha}, \bar{\alpha})$;

4. $\operatorname{dim}_{v} I(\varphi, \psi)=\operatorname{dim}_{v} X$.

Proof. Let $\left\{R_{1}, \ldots, R_{p}\right\}$ be the Markov partition of $X$, and $\chi: \Sigma \rightarrow X$ the associated coding map. The following result is due to Schmeling [10], and constitutes a far-reaching generalization of work of Barreira and Schmeling in [3].

Lemma 9. If $X$ is a repeller of a topologically mixing $C^{1+\varepsilon}$ expanding map, for some $\varepsilon>0$, then $\operatorname{dim}_{v \circ \chi}\left(\chi^{-1} Z\right)=\operatorname{dim}_{v} Z$ for every set $Z \subset X$.

The desired statement is now an immediate consequence of Theorems 48,9 11 and Lemma 9 applied to each of the level sets $K_{\alpha}$. 
Under the hypotheses of Theorem[12, one can also show that if $\alpha \notin(\underline{\alpha}, \bar{\alpha})$, then $K_{\alpha}=\emptyset$, and that if $\varphi$ is cohomologous to some multiple of $\psi$, then $\underline{\alpha}=\bar{\alpha}$.

5.4. Hyperbolic sets. One can also consider diffeomorphisms possessing hyperbolic sets. Let $f: M \rightarrow M$ be a diffeomorphism of a smooth manifold, and $X \subset M$ a compact $f$-invariant set. We say that $X$ is a hyperbolic set of $f$ if there is a continuous splitting of the tangent bundle $T_{X} M=E^{s} \oplus E^{u}$, and constants $\kappa>0$ and $\lambda \in(0,1)$ such that for each $x \in X$ :

1. $d_{x} f E_{x}^{s}=E_{f x}^{s}$ and $d_{x} f E_{x}^{u}=E_{f x}^{u}$;

2. $\left\|d_{x} f^{n} v\right\| \leq \kappa \lambda^{n}\|v\|$ for all $v \in E_{x}^{s}$ and $n \geq 0$;

3. $\left\|d_{x} f^{-n} v\right\| \leq \kappa \lambda^{n}\|v\|$ for all $v \in E_{x}^{u}$ and $n \geq 0$.

The following is a version of Theorem 12 for hyperbolic sets.

Theorem 13. Let $X$ be a locally maximal hyperbolic set of a $C^{1+\varepsilon}$ diffeomorphism $f$, for some $\varepsilon>0$, and $\varphi, \psi, v$ Hölder continuous on $X$ with $\psi>0$ and $v>0$. If $\varphi$ is not cohomologous to any multiple of $\psi$, then:

1. the function $\mathcal{F}_{v}$ is real analytic on $(\underline{\alpha}, \bar{\alpha})$;

2. if $\alpha \in(\underline{\alpha}, \bar{\alpha})$ then

$$
\mathcal{F}_{v}(\alpha)=\max \left\{\frac{h_{\mu}(f)}{\int_{X} v d \mu}: \mu \in \mathcal{M}(X) \text { and } \frac{\int_{X} \varphi d \mu}{\int_{X} \psi d \mu}=\alpha\right\}=\inf _{q \in \mathbb{R}} \Delta(q \alpha, q) \text {, }
$$

where $\Delta(p, q)$ is such that $P_{X}(q \varphi-p \psi-\Delta(p, q) v)=0$;

3. $\inf _{q \in \mathbb{R}} P_{X}\left(q \varphi-q \alpha \psi-\mathcal{F}_{v}(\alpha) v\right)=0$ for every $\alpha \in(\underline{\alpha}, \bar{\alpha})$;

4. $\operatorname{dim}_{v} I(\varphi, \psi)=\operatorname{dim}_{v} X$.

Proof. Locally maximal hyperbolic sets have Markov partitions of arbitrarily small diameter, and each Markov partition has associated a two-sided subshift of finite type $\sigma$, and a coding map $\chi: \Sigma \rightarrow X$ for the hyperbolic set, which is Hölder continuous, onto, and satisfies $f \circ \chi=\chi \circ \sigma$. Furthermore, one can also establish the following version of Lemma 9 for hyperbolic sets (see [10] for details).

Lemma 10. If $X$ is a locally maximal hyperbolic set of a $C^{1+\varepsilon}$ diffeomorphism, for some $\varepsilon>0$, then $\operatorname{dim}_{v \circ \chi}\left(\chi^{-1} Z\right)=\operatorname{dim}_{v} Z$ for every set $Z \subset X$.

The desired statement is now an immediate consequence of Theorems 4 48 11 and Lemma 10 applied to each of the level sets $K_{\alpha}$.

\section{Mixed multifractal SPeCtra}

Let $M$ be a smooth Riemannian manifold and $f: M \rightarrow M$ a $C^{1}$ map. Consider a compact $f$-invariant set $X \subset M$. Assume that $f$ is expanding and that $X$ is a repeller of $f$. We say that $f$ is conformal on $X$ if $d_{x} f$ is a multiple of an isometry for every $x \in X$.

Let $\mathcal{M}(X)$ be the set of $f$-invariant Borel probability measures on $X$, and $\mathcal{M}_{E}(X) \subset \mathcal{M}(X)$ the subset of ergodic measures. Consider a Hölder continuous function $\varphi: X \rightarrow \mathbb{R}$ and define the sets

$$
\begin{gathered}
\mathfrak{D}=\left\{-\frac{\int_{X} \varphi d \mu}{\int_{X} \log \|d f\| d \mu}: \mu \in \mathcal{M}(X)\right\}, \\
\mathfrak{E}=\left\{-\int_{X} \varphi d \mu: \mu \in \mathcal{M}(X)\right\}, \quad \mathfrak{L}=\left\{\int_{X} \log \|d f\| d \mu: \mu \in \mathcal{M}(X)\right\} .
\end{gathered}
$$


When $f$ is a topologically mixing expanding map, each Hölder continuous function $\varphi$ has a unique equilibrium measure which we denote by $\mu_{\varphi}$.

The following statement establishes a conditional variational principle for each

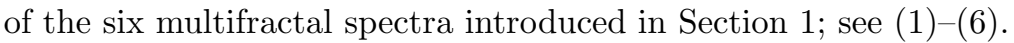

Theorem 14. Let $X$ be a repeller of a topologically mixing $C^{1+\varepsilon}$ expanding map $f$, for some $\varepsilon>0$, such that $f$ is conformal on $X$. If $\varphi$ is a Hölder continuous function with $P_{X}(\varphi)=0$, then:

1. the set $\mathfrak{D}$ is an interval or a point, it coincides with the domains of the functions $\mathcal{D}_{D}^{\mu_{\varphi}}$ and $\mathcal{D}_{E}^{\mu_{\varphi}}$, and if $\alpha \in \operatorname{int} \mathfrak{D}$, then

$$
\begin{aligned}
& \mathcal{D}_{D}^{\mu_{\varphi}}(\alpha)=\max \left\{\operatorname{dim}_{H} \mu: \mu \in \mathcal{M}_{E}(X) \text { and }-\frac{\int_{X} \varphi d \mu}{\int_{X} \log \|d f\| d \mu}=\alpha\right\}, \\
& \mathcal{D}_{E}^{\mu_{\varphi}}(\alpha)=\max \left\{h_{\mu}(f): \mu \in \mathcal{M}_{E}(X) \text { and }-\frac{\int_{X} \varphi d \mu}{\int_{X} \log \|d f\| d \mu}=\alpha\right\} ;
\end{aligned}
$$

2. the set $\mathfrak{E}$ is an interval or a point, it coincides with the domains of the functions $\mathcal{E}_{D}^{\mu_{\varphi}}$ and $\mathcal{E}_{E}^{\mu_{\varphi}}$, and if $\alpha \in$ int $\mathfrak{E}$, then

$$
\begin{aligned}
& \mathcal{E}_{D}^{\mu_{\varphi}}(\alpha)=\max \left\{\operatorname{dim}_{H} \mu: \mu \in \mathcal{M}_{E}(X) \text { and }-\int_{X} \varphi d \mu=\alpha\right\}, \\
& \mathcal{E}_{E}^{\mu_{\varphi}}(\alpha)=\max \left\{h_{\mu}(f): \mu \in \mathcal{M}_{E}(X) \text { and }-\int_{X} \varphi d \mu=\alpha\right\} ;
\end{aligned}
$$

3. the set $\mathfrak{L}$ is an interval or a point, it coincides with the domains of the functions $\mathcal{L}_{D}$ and $\mathcal{L}_{E}$, and if $\alpha \in \operatorname{int} \mathfrak{L}$, then

$$
\begin{aligned}
& \mathcal{L}_{D}(\alpha)=\max \left\{\operatorname{dim}_{H} \mu: \mu \in \mathcal{M}_{E}(X) \text { and } \int_{X} \log \|d f\| d \mu=\alpha\right\}, \\
& \mathcal{L}_{E}(\alpha)=\max \left\{h_{\mu}(f): \mu \in \mathcal{M}_{E}(X) \text { and } \int_{X} \log \|d f\| d \mu=\alpha\right\} .
\end{aligned}
$$

Proof. Recall that when $v=1$ we have $\operatorname{dim}_{v} K=h(f \mid K)$ for any subset $K \subset X$, and $\operatorname{dim}_{v} \mu=h_{\mu}(f)$ for any measure $\mu \in \mathcal{N}(X)$. Furthermore, when $v=\log \|d f\|$ we have $\operatorname{dim}_{v} K=\operatorname{dim}_{H} K$ for any subset $K \subset X$, and $\operatorname{dim}_{v} \mu=\operatorname{dim}_{H} \mu$ for any measure $\mu \in \mathcal{M}(X)$. See Section 2.2 for details.

Let $\left\{R_{1}, \ldots, R_{p}\right\}$ be a Markov partition of $X$, and $\chi: \Sigma \rightarrow X$ the associated coding map. The following is an immediate consequence of Lemma 9

Lemma 11. If $X$ is a repeller of a topologically mixing $C^{1+\varepsilon}$ expanding map $f$, for some $\varepsilon>0$, then $h\left(\sigma \mid \chi^{-1} Z\right)=h(f \mid Z)$ for every set $Z \subset X$. If, in addition, $f$ is conformal on $X$, then $\operatorname{dim}_{v}\left(\chi^{-1} Z\right)=\operatorname{dim}_{H} Z$ for every set $Z \subset X$, where $v=\log \|d f\| \circ \chi$.

Define a function $\psi$ on $X$ by $\psi=\log \|d f\|$. We have

$$
\begin{aligned}
d_{\mu_{\varphi}}(x) & =\lim _{n \rightarrow \infty}-\frac{\varphi_{n}(x)}{\psi_{n}(x)}, \\
h_{\mu_{\varphi}}(x) & =\lim _{n \rightarrow \infty}-\frac{\varphi_{n}(x)}{n}, \\
\lambda(x) & =\lim _{n \rightarrow \infty} \frac{\psi_{n}(x)}{n},
\end{aligned}
$$

whenever the corresponding limits exist. 
By Lemma 11] setting $v=\log \|d f\|$ we obtain each of the first identities in Statements 11 2] and [3, considering respectively the pairs of functions $(\varphi,-\psi)$, $(\varphi,-1)$, and $(\psi, 1)$ in Theorem 6

Again by Lemma 11, setting $v=1$ we obtain each of the second identities in Statements 12 and 3 , considering respectively the pairs of functions $(\varphi,-\psi)$, $(\varphi,-1)$, and $(\psi, 1)$ in Theorem 6

We remark that the conformality hypothesis for $f$ is essential to all but the spectrum $\mathcal{E}_{E}^{\mu_{\varphi}}$. In fact, one can formulate the following stronger statement for this spectrum.

Theorem 15. Let $X$ be a repeller of a topologically mixing $C^{1+\varepsilon}$ expanding map $f$, for some $\varepsilon>0$. If $\varphi$ is a Hölder continuous function with $P_{X}(\varphi)=0$, and $\alpha \in$ int $\mathfrak{E}$ then

$$
\mathcal{E}_{E}^{\mu_{\varphi}}(\alpha)=\max \left\{h_{\mu}(f): \mu \in \mathcal{M}_{E}(X) \text { and }-\int_{X} \varphi d \mu=\alpha\right\} .
$$

Proof. Define the function $\psi=-1$ on $X$. The statement is an immediate consequence of Theorem 5 and Lemma 11 .

The results in Theorems 14 and 15 also follow from the general statement formulated in Theorem 12 .

Under the hypotheses of Theorem 14, it is know that $\mathcal{D}_{D}^{\mu_{\varphi}}$ is analytic on $\mathfrak{D}$, and that $\mathcal{L}_{D}$ and $\mathcal{L}_{E}$ are analytic on $\mathfrak{L}$. Furthermore, under the hypothesis of Theorem [15, it is known that $\mathcal{E}_{E}^{\mu_{\varphi}}$ is analytic on $\mathfrak{E}$. See [7] for references and full details.

It has been a standing open problem in multifractal analysis to decide whether the spectra $\mathcal{D}_{E}$ and $\mathcal{E}_{D}$ are analytic. We shall use the conditional variational principles for $\mathcal{D}_{E}$ and $\mathcal{E}_{D}$ formulated in Theorem 14 to establish that the spectra $\mathcal{D}_{E}$ and $\mathcal{E}_{D}$ are indeed also analytic. We remark that the other four variational principles can also be used to establish the analyticity of the spectra $\mathcal{D}_{D}, \mathcal{E}_{E}, \mathcal{L}_{D}$, and $\mathcal{L}_{E}$. However, as mentioned before, for these four multifractal spectra such a statement is known, and hence, we shall not discuss it any further.

Theorem 16. If $X$ is a repeller of a topologically mixing $C^{1+\varepsilon}$ expanding map $f$, for some $\varepsilon>0$, such that $f$ is conformal on $X$, and $\varphi$ a Hölder continuous function with $P_{X}(\varphi)=0$, then:

1. if $\varphi$ is not cohomologous to a constant, then the function $\mathcal{E}_{D}^{\mu_{\varphi}}$ is real analytic on the non-empty interval $\mathfrak{E}$;

2. if $\varphi$ is not cohomologous to any multiple of $\log \|d f\|$, then the function $\mathcal{D}_{E}^{\mu_{\varphi}}$ is real analytic on the non-empty interval $\mathfrak{D}$.

Proof. Proceeding as in the proof of Theorem 14 the desired statement is an immediate consequence of Statement 2 in Theorem 9

It is well known that under the hypotheses of Theorem 16 there exist a unique invariant probability measure $m_{E}$ of maximal entropy, i.e., such that $h_{m_{E}}(f)=$ $h(f \mid X)$, and a unique invariant probability measure $m_{D}$ of maximal dimension, i.e., such that $\operatorname{dim}_{H} m_{D}=\operatorname{dim}_{H} X$. The measures $m_{E}$ and $m_{D}$ are respectively the equilibrium measures of the functions 0 and $-\operatorname{dim}_{H} X \cdot \log \|d f\|$. Hence, one can reformulate Theorem[16 as follows. 
Theorem 17. If $X$ is a repeller of a $C^{1+\varepsilon}$ expanding map $f$, for some $\varepsilon>0$, such that $f$ is conformal on $X$, and $\mu$ is an equilibrium measure with Hölder continuous potential, then:

1. if $\mu \neq m_{E}$, then the function $\mathcal{E}_{D}^{\mu_{\varphi}}$ is real analytic on $\mathfrak{E}$;

2. if $\mu \neq m_{D}$, then the function $\mathcal{D}_{E}^{\mu_{\varphi}}$ is real analytic on $\mathfrak{D}$.

Furthermore, if $m_{E} \neq m_{D}$ (that is, if $\log \|d f\|$ is not cohomologous to a constant), then for every equilibrium measure $\mu$ with Hölder continuous potential at least one of the spectra $\mathcal{E}_{D}^{\mu_{\varphi}}$ and $\mathcal{D}_{E}^{\mu_{\varphi}}$ is real analytic.

It is known that the multifractal spectra $\mathcal{D}_{D}, \mathcal{E}_{E}, \mathcal{L}_{D}$, and $\mathcal{L}_{E}$ either reduce to a point or are strictly convex (see [7] for details and references). We now illustrate that the mixed multifractal spectra $\mathcal{D}_{E}$ and $\mathcal{E}_{D}$ may in general not be convex. Since Legendre transforms are always convex this implies that the multifractal spectra $\mathcal{D}_{E}$ and $\mathcal{E}_{D}$ in general cannot be expressed as Legendre transforms, with strong contrast with the other four multifractal spectra.

Proposition 18. There exists a repeller of a topologically mixing piecewise linear expanding map of $[0,1]$, and a Bernoulli measure $\mu$ on $[0,1]$, such that the spectrum $\varepsilon_{D}^{\mu}$ is not convex, and hence it is not a Legendre transform.

Construction. For an example with the properties indicated in Proposition 18 one can consider the piecewise linear expanding map $f$ defined on the union of the intervals $I_{0}=\left[0, \mathrm{e}^{-100}\right]$ and $I_{1}=\left[1-\mathrm{e}^{-1}, 1\right]$ such that $f\left(I_{0}\right)=f\left(I_{1}\right)=[0,1]$. Then

$$
X=\bigcap_{k=1}^{\infty} f^{-k}\left(I_{0} \cap I_{1}\right)
$$

is a repeller of $f$. Let $\mu$ be the Bernoulli measure on two symbols such that $\mu\left(I_{0}\right)=$ $1 /(1+\mathrm{e})$ and $\mu\left(I_{1}\right)=\mathrm{e} /(1+\mathrm{e})$. Using the conditional variational principle in Theorem 14 one can explicitly compute $\mathcal{E}_{D}^{\mu}$, and verify that it is not convex; see Proposition 10 above for full details. See Figure 1 for a graph of this spectrum.

A similar construction can be effected for the spectrum $\mathcal{D}_{E}$.

\section{Geometric constructions}

7.1. Non-additive topological pressure. We recall the non-additive version of the topological pressure introduced by Barreira in [1]. We use the notations of Section [2.1.

Let $f: X \rightarrow X$ be a continuous map of a compact metric space, and $\mathcal{U}$ a finite open cover of $X$. Consider a sequence $\Phi$ of functions $\varphi_{n}: X \rightarrow \mathbb{R}$. For each $n \in \mathbb{N}$ let

$$
\gamma_{n}(\Phi, \mathcal{U})=\sup \left\{\left|\varphi_{n}(x)-\varphi_{n}(y)\right|: x, y \in X(\mathbf{U}) \text { for some } \mathbf{U} \in \mathcal{W}_{n}(\mathcal{U})\right\}
$$

We assume that

$$
\limsup _{\operatorname{diam} \mathcal{U} \rightarrow 0} \limsup _{n \rightarrow \infty} \frac{\gamma_{n}(\Phi, \mathcal{U})}{n}=0 .
$$

Given $\mathbf{U} \in \mathcal{W}_{n}(\mathcal{U})$ we set

$$
\Phi(\mathbf{U})= \begin{cases}\sup _{X(\mathbf{U})} \varphi_{n} & \text { if } X(\mathbf{U}) \neq \emptyset \\ -\infty & \text { if } X(\mathbf{U})=\emptyset\end{cases}
$$


For each set $Z \subset X$ and number $\alpha \in \mathbb{R}$, let

$$
M(Z, \alpha, \Phi, \mathcal{U})=\lim _{n \rightarrow \infty} \inf _{\Gamma} \sum_{U \in \Gamma} \exp (-\alpha m(U)+\Phi(\mathbf{U})),
$$

where the infimum is taken over all $\Gamma \subset \bigcup_{k \geq n} \mathcal{W}_{n}(\mathcal{U})$ covering $Z$. We define the non-additive topological pressure of the sequence of functions $\Phi$ on the set $Z$ (with respect to $f$ ) by

$$
P_{Z}(\Phi)=\lim _{\operatorname{diam} u \rightarrow 0} \inf \{\alpha: M(Z, \alpha, \Phi, \mathcal{u})=0\} .
$$

When $\Phi$ is the sequence of functions $\varphi_{n}=\sum_{k=0}^{n-1} \varphi \circ f^{k}$, for some continuous function $\varphi: X \rightarrow \mathbb{R}$, the assumption (18) is satisfied, and $P_{X}(\Phi)$ coincides with the topological pressure $P_{X}(\varphi)$ introduced in Section 2.1

The non-additive thermodynamic formalism has been applied with success to a number of problems in dimension theory of dynamical systems; see [1, 7] for details.

7.2. Formulation of the results. We can also establish a non-additive version of Theorem 8. Consider three sequences of functions $\Phi=\left\{\varphi_{n}\right\}, \Psi=\left\{\psi_{n}\right\}$, and $\Upsilon=\left\{v_{n}\right\}$ on the space $X$. We assume that $\Phi, \Psi$, and $\Upsilon$ satisfy hypothesis (18). Consider the set

$$
L_{\alpha}=\left\{x \in X: \lim _{n \rightarrow \infty} \frac{\varphi_{n}(x)}{\psi_{n}(x)}=\alpha\right\} .
$$

Theorem 19. If $\sup \left\{\left|\psi_{n}(x)\right| / n: x \in X, n \in \mathbb{N}\right\}<\infty$, then

$$
P_{L_{\alpha}}(\Upsilon) \leq \inf _{q \in \mathbb{R}} P_{X}(q \Phi-q \alpha \Psi+\Upsilon) .
$$

Proof. This is a modification of the proof of Lemma 1. By defining sets $L_{\delta, \tau}$ as in the proof of Lemma 1 and using similar arguments we conclude that

$$
P_{L_{\delta, \tau}}(\Upsilon, \mathcal{U}) \leq P_{L_{\delta, \tau}}(q(\Phi-\alpha \Psi)+\Upsilon, \mathcal{U})+(2+|\alpha|) \delta|q| .
$$

Letting the diameter of $\mathcal{U}$ going to zero yields

$$
P_{L_{\delta, \tau}}(\Upsilon) \leq P_{X}(q \Phi-q \alpha \Psi+\Upsilon)+(2+|\alpha|) \delta|q|,
$$

and hence,

$$
P_{L_{\alpha}}(\Upsilon) \leq \sup _{\tau \in \mathbb{N}} P_{L_{\delta, \tau}}(\Upsilon) \leq P_{X}(q \Phi-q \alpha \Psi+\Upsilon)+(2+|\alpha|) \delta|q| .
$$

Since $\delta$ is arbitrary, we obtain $P_{L_{\alpha}}(\Upsilon) \leq P_{X}(q \Phi-q \alpha \Psi+\Upsilon)$.

Taking $\Upsilon=0$ in Theorem 19 one obtains the following.

Theorem 20. Under the hypotheses of Theorem 19, we have

$$
h\left(f \mid L_{\alpha}\right) \leq \inf _{q \in \mathbb{R}} P_{X}(q \Phi-q \alpha \Psi) .
$$

This provides a non-additive generalization of the identity (12). Theorem 20 can be used to establish the foundations of a multifractal analysis for limit sets of geometric constructions. Here we shall only discuss a first application. Further topics will be discussed elsewhere.

A symbolic geometric construction in $\mathbb{R}^{m}$ is defined by:

1. a compact set $Q \subset\{1, \ldots, p\}^{\mathbb{N}}$ which is invariant under the shift map $f$ : $\{1, \ldots, p\}^{\mathbb{N}} \rightarrow\{1, \ldots, p\}^{\mathbb{N}}$ 
2. a decreasing sequence of compact sets $\Delta_{i_{1} \cdots i_{n}} \subset \mathbb{R}^{m}$ for each sequence $\left(i_{1} i_{2} \cdots\right)$ $\in Q$, with diameter diam $\Delta_{i_{1} \cdots i_{n}} \rightarrow 0$ as $n \rightarrow \infty$.

The limit set of the construction is the compact set

$$
F=\bigcap_{n \in \mathbb{N}} \bigcup_{i_{1} \cdots i_{n}} \Delta_{i_{1} \cdots i_{n}}
$$

where the union is taken over all tuples $\left(i_{1} \cdots i_{n}\right)$ such that $\left(i_{1} \cdots i_{n}\right)=\left(j_{1} \cdots j_{n}\right)$ for some $\left(j_{1} \cdots\right) \in Q$. We notice that these constructions include as trivial examples any iterated function systems defined by a family of contraction maps.

Consider the sequence of functions $\varphi_{n}\left(i_{1} i_{2} \cdots\right)=\log \operatorname{diam} \Delta_{i_{1} \cdots i_{n}}$ on $Q$, and denote it $\Phi$. It is easy to verify that $\Phi$ satisfies hypothesis (18). We assume that:

1. the set $\Delta_{i_{1} \cdots i_{n}}$ is a ball of diameter $d_{i_{1} \cdots i_{n}}$;

2. $\Delta_{i_{1} \cdots i_{n}} \cap \Delta_{j_{1} \cdots j_{n}}=\emptyset$ whenever $\left(i_{1} \cdots i_{n}\right) \neq\left(j_{1} \cdots j_{n}\right)$;

3. $\varphi_{n+m} \leq \varphi_{n}+\varphi_{m} \circ f^{n}$ for every $n, m \in \mathbb{N}$;

4. $\sup \left\{\varphi_{n}(\omega)-\varphi_{n+1}(\omega): \omega \in Q\right.$ and $\left.n \in \mathbb{N}\right\}<\infty$.

We notice that the numbers $d_{i_{1} \cdots i_{n}}$ may depend on all the symbolic past, and may satisfy no asymptotic behavior. It follows from [1, Theorem 2.1] that under those hypotheses, we have $\operatorname{dim}_{H} F=s$, where $s$ is the unique number such that $P_{Q}(s \Phi)=0$.

The following is an immediate consequence of Theorem 20 .

Theorem 21. We have

$$
h\left(f \mid\left\{\left(i_{1} i_{2} \cdots\right) \in Q: \lim _{n \rightarrow \infty}-\frac{\log d_{i_{1} \cdots i_{n}}}{n}=\alpha\right\}\right) \leq \inf _{q \in \mathbb{R}}\left(P_{Q}(q \Phi)+q \alpha\right) .
$$

This statement establishes an upper bound for the entropy spectrum of Lyapunov exponents associated with the geometric construction. Notice that the upper bound is a Legendre transform involving the non-additive topological pressure. It is an open problem to obtain a sharp lower bound.

\section{ACKNOWLEDGMENT}

We would like to thank the referee for the careful reading of our paper and for a good number of suggestions.

\section{REFERENCES}

1. L. Barreira, A non-additive thermodynamic formalism and applications to dimension theory of hyperbolic dynamical systems, Ergodic Theory Dynam. Systems 16 (1996), 871-927. MR 98a:58124

2. L. Barreira, Ya. Pesin and J. Schmeling, Dimension and product structure of hyperbolic measures, Ann. of Math. (2) 149 (1999), 755-783. MR 2000f:37027

3. L. Barreira and J. Schmeling, Sets of "non-typical" points have full topological entropy and full Hausdorff dimension, Israel J. Math. 116 (2000), 29-70. CMP 2000:13

4. R. Bowen, Some systems with unique equilibrium states, Math. Systems Theory 8 (1975), 193-202. MR 53:3257

5. G. Keller, Equilibrium states in ergodic theory, London Mathematical Society Student Texts 42, Cambridge University Press, 1998. MR 99e:28022

6. E. Olivier, Analyse multifractale de fonctions continues, C. R. Acad. Sci. Paris 326 (1998), 1171-1174. MR 99h:58109

7. Ya. Pesin, Dimension theory in dynamical systems: contemporary views and applications, Chicago Lectures in Mathematics, Chicago University Press, 1997. MR 99b:58003

8. D. Ruelle, Thermodynamic formalism, Encyclopedia of mathematics and its applications, vol. 5, Addison-Wesley, 1978. MR 80g:82017 
9. J. Schmeling, Symbolic dynamics for $\beta$-shifts and self-normal numbers, Ergodic Theory Dynam. Systems 17 (1997), 675-694. MR 98e:11080

10. J. Schmeling, Entropy preservation under Markov coding, preprint.

11. F. Takens and E. Verbitski, On the variational principle for the topological entropy of certain non-compact sets, preprint.

12. P. Walters, Equilibrium states for $\beta$-transformations and related transformations, Math. Z. 159 (1978), 65-88. MR 57:6370

Departamento de Matemática, Instituto Superior Técnico, 1049-001 Lisboa, Portugal

E-mail address: luis.barreira@math.ist.utl.pt

URL: http://www.math.ist.utl.pt/ ’barreira/

LAmFa / CNRS Fre 2270, Université de Picardie Jules Verne, 33 rue Saint Leu, 80039 Amiens, France

E-mail address: benoit.saussol@mathinfo.u-picardie.fr

URL: http://www.mathinfo.u-picardie.fr/saussol/ 